\title{
Calibration of Ultraviolet, Mid-infrared, and Radio Star Formation Rate Indicators
}

\author{
Michael J. I. Brown ${ }^{1,2}{ }^{(10}$, John Moustakas ${ }^{3}$, Robert C. Kennicutt ${ }^{4}$, Nicolas J. Bonne ${ }^{5}\left(\mathbb{D}\right.$, Huib T. Intema ${ }^{6}{ }^{(1)}$,

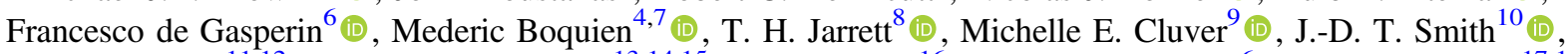 \\ Elisabete da Cunha ${ }^{11,12}$ (D), Masatoshi Imanishi ${ }^{13,14,15}$ (D), Lee Armus ${ }^{16}$, Bernhard R. Brandl ${ }^{6}$, and J. E. G. Peek ${ }^{17,18}{ }^{(D)}$ \\ ${ }^{1}$ School of Physics and Astronomy, Monash University, Clayton, Victoria 3800, Australia; Michael.Brown@monash.edu \\ ${ }^{2}$ Monash Centre for Astrophysics, Monash University, Clayton, Victoria, 3800, Australia \\ ${ }^{3}$ Department of Physics and Astronomy, Siena College, 515 Loudon Road, Loudonville, NY 12211, USA \\ ${ }^{4}$ Institute of Astronomy, University of Cambridge, Cambridge, CB3 OHA, UK \\ ${ }^{5}$ Institute for Cosmology and Gravitation, Dennis Sciama Building, University of Portsmouth, Burnaby Road, Portsmouth PO1 3FX, UK \\ ${ }^{6}$ Leiden Observatory, Leiden University, P.O. Box 9513, 2300 RA Leiden, The Netherlands \\ ${ }^{7}$ Universidad de Antofagasta, Unidad de Astronomia, Avenida Angamos 601, 02800 Antofagasta, Chile \\ ${ }^{8}$ Astrophysics, Cosmology and Gravity Centre (ACGC), Astronomy Department, University of Cape Town, Private Bag X3, Rondebosch 7701, South Africa \\ ${ }^{9}$ University of the Western Cape, Robert Sobukwe Road, Bellville 7535, South Africa \\ ${ }^{10}$ Department of Physics and Astronomy, University of Toledo, Ritter Obs., MS \#113, Toledo, OH 43606, USA \\ ${ }^{11}$ Research School of Astronomy and Astrophysics, Australian National University, Canberra, ACT 2611, Australia \\ ${ }^{12}$ Centre for Astrophysics and Supercomputing, Swinburne University of Technology, Hawthorn, Victoria 3122, Australia \\ ${ }^{13}$ Subaru Telescope, 650 North A'ohoku Place, Hilo, HI 96720, USA \\ ${ }^{14}$ Department of Astronomy, School of Science, Graduate University for Advanced Studies (SOKENDAI), Mitaka, Tokyo 181-8588, Japan \\ ${ }^{15}$ National Astronomical Observatory of Japan, 2-21-1 Osawa, Mitaka, Tokyo 181-8588, Japan \\ ${ }^{16}$ Spitzer Science Center, California Institute of Technology, Pasadena, CA, USA \\ ${ }^{17}$ Space Telescope Science Institute, 3700 San Martin Drive, Baltimore, MD 21218, USA \\ ${ }^{18}$ Department of Astronomy, Columbia University, NY, USA \\ Received 2016 November 24; revised 2017 August 5; accepted 2017 August 31; published 2017 October 3
}

\begin{abstract}
We present calibrations for star formation rate (SFR) indicators in the ultraviolet, mid-infrared, and radiocontinuum bands, including one of the first direct calibrations of $150 \mathrm{MHz}$ as an SFR indicator. Our calibrations utilize 66 nearby star-forming galaxies with Balmer-decrement-corrected $\mathrm{H} \alpha$ luminosities, which span five orders of magnitude in SFR and have absolute magnitudes of $-24<M_{r}<-12$. Most of our photometry and spectrophotometry are measured from the same region of each galaxy, and our spectrophotometry has been validated with SDSS photometry, so our random and systematic errors are small relative to the intrinsic scatter seen in SFR indicator calibrations. We find that the Wide-field Infrared Space Explorer W4 (22.8 $\mu \mathrm{m})$, Spitzer $24 \mu \mathrm{m}$, and $1.4 \mathrm{GHz}$ bands have tight correlations with the Balmer-decrement-corrected $\mathrm{H} \alpha$ luminosity, with a scatter of only 0.2 dex. Our calibrations are comparable to those from the prior literature for $L^{*}$ galaxies, but for dwarf galaxies, our calibrations can give SFRs that are far greater than those derived from most previous literature.
\end{abstract}

Key words: dust, extinction - galaxies: evolution - galaxies: general - galaxies: photometry - stars: formation techniques: spectroscopic

Supporting material: machine-readable table

\section{Introduction}

Galaxies increase their stellar masses via star formation and mergers, and thus measurements of galaxy star formation rates (SFRs) are critical for many observational studies of galaxy evolution. In principle, very accurate SFRs are provided by ultraviolet and hydrogen recombination line luminosities, which directly trace the population of short-lived very massive stars (Kennicutt \& Evans 2012 and references therein). In practice, measured ultraviolet luminosities are sensitive to dust attenuation, and accurate spectrophotometry is often unavailable or limited to the cores of galaxies. For example, the vast majority of galaxies in deep optical, mid-infrared, and radiocontinuum surveys do not have spectroscopic redshifts, and this will remain true for the foreseeable future (e.g., The Dark Energy Survey Collaboration 2005; Papovich et al. 2006; Norris et al. 2011).

As a consequence of the limitations of spectroscopy and ultraviolet imaging, a number of SFR indicators have been utilized at mid-infrared, far-infrared, and radio wavelengths. For a detailed discussion of these SFR indicators and their calibration, we refer the reader to Kennicutt et al. (2009),
Kennicutt \& Evans (2012), and references therein. The integrated far-infrared emission is (comparatively) straightforward to understand, as it results from dust heated primarily by ultraviolet and optical photons from massive stars. However, at specific wavelengths, the emission has a nontrivial relationship with the SFR. For example, measurements with the Wide-field Infrared Space Explorer (WISE) W3 (12 $\mu \mathrm{m})$ band can include contributions from thermal emission from dust, a deep silicate absorption feature, and emission attributed to polycyclic aromatic hydrocarbons (PAHs), which has a metallicity dependence (e.g., Houck et al. 2004; Engelbracht et al. 2005, 2008; Jackson et al. 2006; Draine et al. 2007; Smith et al. 2007). Furthermore, the thermal emission from dust can result from star formation, active galactic nuclei (AGNs), and old stellar populations (e.g., Walterbos \& Schwering 1987; Bendo et al. 2010; Boquien et al. 2011). It is possible to model the relationship between the observed galaxy luminosities and SFRs via detailed galaxy spectral energy distribution (SED) modeling (e.g., da Cunha et al. 2008; Boquien et al. 2016; Davies et al. 2016; Leja et al. 2017), but a more common approach is to empirically calibrate SFR indicators using 
hydrogen recombination line luminosities with corrections for dust attenuation.

Although empirical calibrations of SFR indicators are far simpler than SED modeling, they are not completely free from modeling and the resulting model-dependent assumptions. The relationship between $\mathrm{H} \alpha$ luminosity and SFR depends on the adopted stellar initial mass function (IMF), which may not be universal (e.g., van Dokkum \& Conroy 2010), and the recent star formation history (e.g., Weisz et al. 2012; da Silva et al. 2014). Dust obscuration is often modeled using a dusty screen rather than more complex (and realistic, yet uncertain) dust geometries, and the Balmer decrement measurements of dust obscuration typically adopt a set of conditions for the interstellar gas that cannot apply throughout individual galaxies, let alone throughout entire galaxy populations (e.g., Calzetti et al. 1994; Boquien et al. 2012, and references therein). Measurements of weak nebular emission lines in galaxy spectra rely on subtracting the stellar continuum, which requires modeling of star formation histories and stellar populations (including details such as metallicity). Relationships between the SFR indicator and hydrogen recombination line luminosities are frequently modeled with linear relationships or power laws, without clear physical motivation (although good fits can be achieved). That said, as discussed by Kennicutt et al. (2009), such simplified (and transparent) modeling can still produce reliable calibrations for SFR indicators consistent with the more complicated modeling of galaxy SEDs.

The empirical calibrations of SFR indicators are critically reliant on the accuracy of measurements of hydrogen recombination line fluxes, dust attenuation corrections, and photometry, all of which present challenges. Achieving spectrophotometric accuracies better than $10 \%$ is nontrivial, and spectroscopy is often limited to galaxy cores (e.g., fiber-fed and slit spectroscopy), requiring aperture corrections to measure hydrogen recombination line fluxes for entire galaxies (e.g., Hopkins et al. 2003; Brough et al. 2011). Matching catalogs of emission-line fluxes and catalogs of broadband photometry can be performed relatively quickly, but ideally spectra and photometry should be extracted from the same regions of individual galaxies (thus mitigating difficulties with aperture corrections). Reliable emission-line fluxes require the accurate subtraction of the continuum and absorption lines from stellar populations (e.g., Tremonti et al. 2004; Moustakas $\&$ Kennicutt 2006), and Balmer decrement corrections of dust attenuation require high signal-to-noise measurements of emission lines. Photometric zero-point errors, effective wavelength errors, and other systematic errors (e.g., scattered light in the Spitzer IRAC detector) can hamper the calibration of SFR indicators. For example, in Brown et al. (2014b), we identified an effective wavelength error in the WISE W4 filter curve, which results in the $22 \mu \mathrm{m}$ flux densities of luminous infrared galaxies (LIRGs) being overestimated by up to $30 \%$.

Sample selection inevitably plays a role in SFR indicator calibrations. Magnitude-limited samples are dominated by $\sim L^{*}$ galaxies that fall on the SFR-mass relation (i.e., the "starforming main sequence," Noeske et al. 2007) and have relatively few low-luminosity dwarf galaxies and LIRGs. Many galaxy samples have minimum redshift, maximum size (e.g., for integral field or fiber-fed spectroscopy), and maximum flux limits (e.g., to prevent cross-talk in multi-object spectroscopy), which effectively place limits on galaxy stellar masses and SFRs. For example, the Cluver et al. (2014) calibration of the WISE W3 and W4 bands uses galaxies with SFRs greater than $10^{-1} M_{\odot} \mathrm{yr}^{-1}$. Consequently, a number of the SFR calibrations from the literature use samples with $\mathrm{H} \alpha$ luminosities that span less than three orders of magnitude $(\mathrm{Wu}$ et al. 2005; Lee et al. 2013; Cluver et al. 2014; CatalánTorrecilla et al. 2015), and extrapolations of such empirical calibrations obviously carry risks.

SFR indicator calibrations have been extended to low SFRs using individual H II regions, but the relationship between the SFR indicator luminosity and the SFR of $\mathrm{H}$ II regions in $\sim L^{*}$ galaxies differs from that in dwarf galaxies (e.g., Calzetti et al. 2007; Relaño et al. 2007; Kennicutt et al. 2009). Prior to the widespread availability of Spitzer and WISE mid-infrared archival imaging, Infrared Astronomical Satellite (IRAS) photometry was used for mid-infrared SFR calibrations, which excludes low-luminosity galaxies and potentially introduces errors when IRAS fluxes are used as proxies for Spitzer and WISE fluxes (Kennicutt et al. 2009). Of course, these issues are well-known to the relevant authors, who were generally using the best available data at the time of publication.

In this paper, we present SFR calibrations for the Galaxy Evolution Explorer (GALEX) FUV band, Spitzer mid-infrared bands, WISE mid-infrared bands and radio continuum. Our focus is on monochromatic SFR indicators, in part due to the data we currently have available and in part because such calibrations will be readily usable by new deep wide-field surveys (e.g., Norris et al. 2011; Williams et al. 2016). The calibrations utilize the photometry and SEDs of Brown et al. (2014b), and new photometry of galaxies with distances of $\lesssim 10 \mathrm{Mpc}$. The bulk of the photometry and spectrophotometry is accurate to $10 \%$, and for most wavelengths, our photometry and spectra are extracted from the same region of each galaxy, minimizing the impact of aperture corrections. Our galaxy sample spans $-24<M_{r}<$ -12 and $-0.3<u-r<2.3$ (AB), and includes LIRGs and blue compact dwarfs, as well as regular $\sim L^{*}$ spiral galaxies. Balmer-decrement-corrected $\mathrm{H} \alpha$ luminosities, and thus SFRs, span almost five orders of magnitude. We thus expect our SFR indicator calibrations to be applicable to a broader range of galaxies than many of the calibrations from previous literature.

The structure of this paper is as follows. Section 2 presents an overview of the archival imaging, photometry, and spectroscopy used in our study. In Section 3, we discuss our new emission-line flux measurements, which are critical for sample selection and Balmer decrement $\mathrm{H} \alpha$ luminosity measurements. In Section 4, we describe the selection of the star-forming galaxy sample and the basic observable properties of this sample (e.g., absolute magnitudes, colors). The calibration of SFR indicators is discussed in Section 5, and our principal conclusions are summarized in Section 6 . Throughout this paper, we use $\mathrm{AB}$ magnitudes and adopt a bolometric luminosity of $3.827 \times 10^{33} \mathrm{erg} \mathrm{s}^{-1}$ for the Sun. To simplify comparison with previous literature, broadband luminosities are $\nu L_{\nu}$ with units of $\mathrm{erg} \mathrm{s}^{-1}$, while radio powers are presented in units of $\mathrm{W} \mathrm{Hz}^{-1}$.

\section{Data}

Our parent sample is star-forming galaxies with optical driftscan spectrophotometry from Moustakas \& Kennicutt (2006) and Moustakas et al. (2010) that also have Sloan Digital Sky 
Survey III optical imaging (SDSS-III; Aihara et al. 2011). The extraction apertures for the optical spectrophotometry vary in size between $20^{\prime \prime} \times 20^{\prime \prime}$ and $\sim 15^{\prime} \times 3^{\prime}$, and thus the spectra include much of the relevant galaxy light. We presented the ultraviolet to mid-infrared photometry and SEDs for many of these galaxies in Brown et al. (2014b). For the galaxies that were not previously presented in Brown et al. (2014b), the data sources and methods are effectively identical to those of Brown et al. (2014b).

All of the galaxies in the sample have imaging at ultraviolet, optical, near-infrared, and mid-infrared wavelengths, taken from GALEX (Morrissey et al. 2007), the Swift UV/Optical Monitor Telescope (Roming et al. 2005), the Sloan Digital Sky Survey III (SDSS-III; Aihara et al. 2011), the Two Micron All Sky Survey (2MASS; Skrutskie et al. 2006), the Spitzer Space Telescope (Fazio et al. 2004; Rieke et al. 2004), and/or the Wide-field Infrared Space Explorer (WISE; Wright et al. 2010). Absolute photometric calibration for these imaging surveys is typically on the order of a few percent for stellar sources (Skrutskie et al. 2006; Padmanabhan et al. 2008; Wright et al. 2010; Bohlin et al. 2011, 2014), although larger photometric calibration errors may be present in the UV (GALEX calibration issues are discussed in detail by Camarota \& Holberg 2014) and for extended source photometry (e.g., Jarrett et al. 2011). Foreground dust extinction was modeled using the Planck dust-extinction maps (Planck Collaboration et al. 2011, 2014) and the Fitzpatrick (1999) extinction curve, with the modification to the UV attenuation proposed by Peek (2013). However, it should be noted that for the bulk of the galaxies in our sample, the foreground dust extinction is less than $E(B-V)=0.05$.

Matched aperture photometry was measured in all bands shortward of $30 \mu \mathrm{m}$ using the same rectangular aperture that was used for the optical drift-scan spectrophotometry. The methods used to measure the aperture photometry are largely identical to those of Brown et al. (2014b), including coincidence loss corrections for Swift photometry and scattered light corrections for Spitzer IRAC photometry. However, unlike Brown et al. (2014b), we corrected for the difference between the in-orbit and laboratory measured WISE W4 effective wavelengths, using the method of Brown et al. (2014a). Uncertainties were determined by measuring aperture photometry at positions offset from the galaxy position and then measuring the range that encompassed $68 \%$ of the data. For most galaxies and bands, the uncertainties are less than $0.1 \mathrm{mag}$, and for the SFR calibrations, we exclude photometry if the uncertainties are greater than $0.2 \mathrm{mag}$.

All galaxies in the Brown et al. (2014b) sample with WISE colors of $W 2-W 3 \gtrsim 0$ (i.e., significant mid-infrared emission from warm dust) have low-resolution 5-38 $\mu \mathrm{m}$ spectra from the Spitzer Infrared Spectrograph (IRS). The requirement for IRS spectra for star-forming galaxies was one of the biggest limitations on the Brown et al. (2014b) sample size, and effectively excluded low-luminosity dwarf galaxies from that sample. To correct for this weakness and extend our SFR calibration to low luminosities, we have added galaxies to the sample that have Moustakas \& Kennicutt (2006) and Moustakas et al. (2010) drift-scan spectrophotometry, SDSSIII imaging, and distances of less than $10 \mathrm{Mpc}$. Photometry for these galaxies was measured in the same bands as the Brown et al. (2014b) sample (when available), and the optical color-

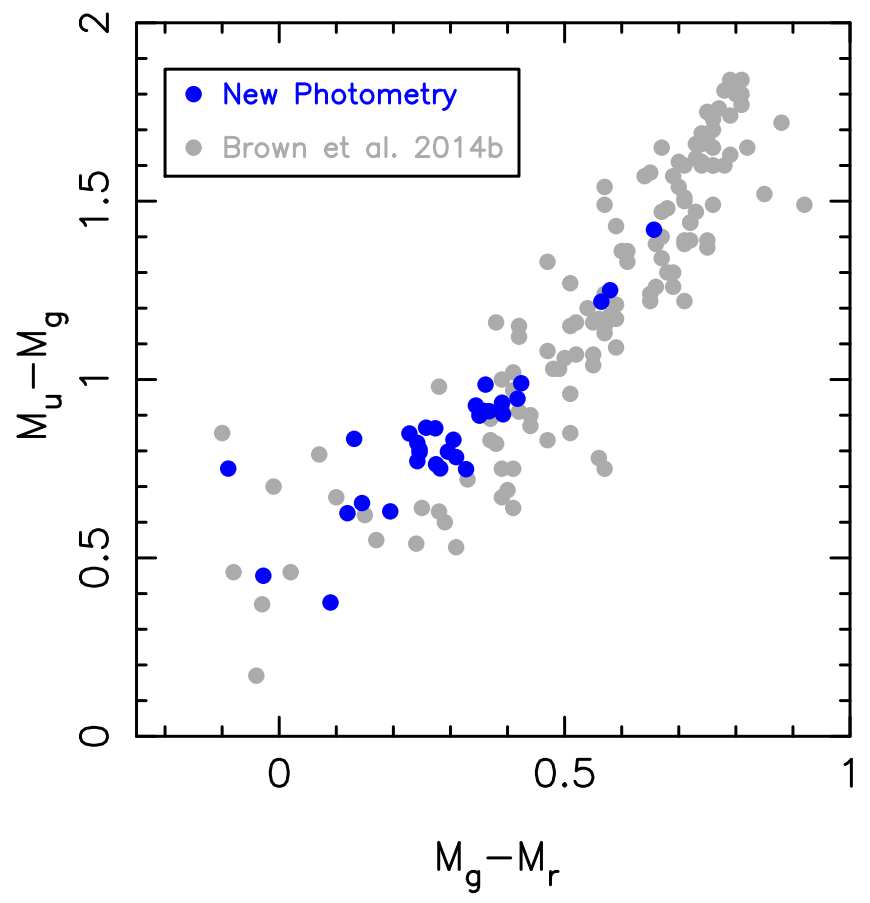

Figure 1. Photometry of the Brown et al. (2014b) sample galaxies and galaxies from Moustakas \& Kennicutt (2006) and Moustakas et al. (2010) with distances of less than $10 \mathrm{Mpc}$. As the photometric uncertainties are typically less than $0.1 \mathrm{mag}$, for the sake of clarity we did not include uncertainties in this plot (and this is the case for most plots in this paper). Unsurprisingly, the addition of nearby galaxies increases the number of blue low-metallicity dwarfs in the sample.

color diagram of the expanded sample of 161 galaxies is presented in Figure 1.

For each galaxy, the spectrophotometry was renormalized by a factor determined by dividing SDSS $g$-band aperture photon fluxes with $g$-band photon fluxes synthesized from the spectra. This resulted in systematic increases in the continuum and emission-line fluxes of roughly $10 \%$, with larger corrections being common for galaxies brighter than $m_{g}=12$. Calibration of drift-scan spectrophotometry is nontrivial (i.e., Moustakas \& Kennicutt 2006; Kennicutt et al. 2008), and for the brightest galaxies, oversubtraction of the sky background may have enhanced the systematic errors.

We expect some of the relationships presented in this paper to depend on the total galaxy luminosity (or galaxy stellar mass), and these relationships can be nonlinear. As a consequence, when calibrating SFR indicators, we rescaled the broadband and emission-line aperture fluxes by a factor equal to the $g$-band total flux divided by the $g$-band aperture flux. (This rescaling differs from a typical aperture-bias correction, which accounts for broadband and emission-line fluxes being measured using apertures of different sizes.) For most galaxies, the total magnitude was the brighter of the aperture magnitude or the magnitude provided by the NASASloan Atlas (Blanton et al. 2011). For some galaxies where the aperture is smaller than the galaxy size and the NASA Sloan Atlas magnitude is absent or in error, we remeasured the "total" magnitudes using large-aperture photometry. ${ }^{19}$

\footnotetext{
${ }^{19}$ We remeasured total magnitudes for Mrk 33, NGC 337, NGC 628, NGC 2403, NGC 3049, NGC 3198, NGC 3351, NGC 3521, NGC 3627 , NGC 4254, NGC 4559, NGC 4569, NGC 4656, NGC 4631, NGC 4670, and NGC 5055.
} 
Radio-continuum flux densities at $1.4 \mathrm{GHz}$ and $150 \mathrm{MHz}$ were determined using multiple sets of archival data. Our principal source of $1.4 \mathrm{GHz}$ flux densities is the NRAO VLA Sky Survey (NVSS; Condon et al. 1998), which has an angular resolution of $45^{\prime \prime}$ and an rms of $0.45 \mathrm{mJy}$ per beam. The NVSS flux calibration is tied to the Baars et al. (1977) absolute scale, and for compact sources, NVSS flux densities agree with those of the Westerbork/Einstein surveys to within a few percent (Condon et al. 1998). Most of our galaxies have counterparts in the default NVSS catalog, but when available, we used the flux densities from Condon et al. (2002), which include single-dish flux densities for the brightest radio sources. A small number of galaxies have no cataloged NVSS flux densities and are relatively compact in size (less than $60^{\prime \prime}$ by $60^{\prime \prime}$ ), and for these galaxies we measured point-source flux densities from the NVSS maps at the galaxy positions.

Our principal source of $150 \mathrm{MHz}$ flux densities is the TIFR GMRT Sky Survey (TGSS; e.g., Bagchi et al. 2011; GopalKrishna et al. 2012; Sirothia et al. 2014), which has an angular resolution of $\sim 25^{\prime \prime}$ and an rms of $\sim 3.5 \mathrm{mJy}$ per beam. We used the first alternative data release of the TGSS (TGSS ADR1; Intema et al. 2017), which provides images and catalogs for nearly the full TGSS survey area. TGSS ADR1 flux densities are tied to the Scaife \& Heald (2012) scale, while comparisons with other surveys show TGSS flux densities for bright compact radio sources are 5\% brighter than the Seventh Cambridge Survey of Radio Sources flux densities and almost identical to LOFAR flux densities (Intema et al. 2017).

To measure the TGSS flux densities for our galaxies, we defined elliptical apertures that encompassed the vast majority of the galaxy light identified in optical, mid-infrared, and TGSS images. We then measured the flux densities directly from copies of the TGSS images with a reduced angular resolution of $\sim 45^{\prime \prime}$, which improves the detectability of the extended emission. The TGSS ADR1 is optimized for imaging of compact sources, and therefore becomes less reliable for measuring flux densities for galaxies larger than a few arcminutes. For the brightest radio sources in our sample, we used flux densities from the Sixth and Seventh Cambridge Surveys of Radio Sources (6C and 7C; Baldwin et al. 1985; Hales et al. 1988, 1990, 1991, 1993a, 1993b, 2007) and the GaLactic and Extragalactic All-Sky MWA Survey (GLEAM; Wayth et al. 2015; Hurley-Walker et al. 2017), which do not have the angular size limitations of the TGSS but are more prone to source confusion. Changes to the selection criteria used for radio flux density measurements (e.g., the criteria used to exclude large galaxies) had little impact on our SFR indicator calibrations.

As the relationships between SFR and luminosity can be nonlinear, and many of our galaxies have distances of less than $10 \mathrm{Mpc}$, we utilize redshift-independent distances (when available) or distances corrected for cosmic flows. Our sources of redshift-independent distances are Tully et al. (2013) and Sorce et al. (2014), with the exception of NGC 4569 and UGCA 166, where we use distances from Cortés et al. (2008) and Marconi et al. (2010), respectively. For the nearest starforming galaxies, redshift-independent distances are primarily from the tip of the red giant branch and Cepheids, although beyond $10 \mathrm{Mpc}$ most redshift-independent distances are derived from the Tully-Fisher relation. For the 72 galaxies without redshift-independent distances, we use distances that account for cosmic flows induced by Virgo, the Shapley supercluster, and the Great Attractor, using the prescription of Mould et al. (2000). Distance errors do not impact calibrations where the SFR indicator luminosity is directly proportional to the SFR. However, if the relationship between the luminosity and SFR is a power law with an index of 1.3, then a distance error of $20 \%$ will translate to luminosity and SFR errors of $44 \%$, resulting in an offset from the power-law relation of 0.05 dex. This offset is relatively small, so we expect distance errors to have little impact on our SFR indicator calibrations.

\section{Emission-Line Fluxes}

A significant change for this paper relative to previous studies using the Moustakas \& Kennicutt (2006) and Moustakas et al. (2010) spectra is revised emission-line fluxes. In order to minimize the systematic differences in the emission-line fluxes from these two sources, we remeasured in a consistent way the strong nebular lines from the original flux-calibrated spectra. Following Moustakas et al. (2011), we used modified versions of pPXF $^{20}$ (Cappellari \& Emsellem 2004) and GANDALF ${ }^{21}$ (Sarzi et al. 2006) to model the stellar continuum and nebular emission lines, respectively. We fitted each stellar spectrum (after masking the emission lines) using a non-negative linear combination of 10 Solar-metallicity Bruzual \& Charlot (2003) population synthesis models with instantaneous-burst ages ranging from $5 \mathrm{Myr}$ to 13 Gyr, assuming a Chabrier (2003) IMF from 0.1 to $100 M_{\odot}$.

The fitting was executed twice, once using cross-correlation to allow for small adjustments to the fiducial redshift and a second time keeping the redshift fixed and fitting the continuum simultaneously with the stellar velocity dispersion. We treated the selective extinction $E(B-V)$ as a free parameter for all stellar ages and attenuate each spectrum using the Calzetti et al. (2000) dust law. We verified that altering several of these assumptions had a negligible effect on our results: allowing a wider range of both sub- and super-solar stellar metallicities; including a larger number of instantaneousburst ages; adopting a different dust law (e.g., O'Donnell 1994); or allowing for time-dependent extinction (e.g., Charlot \& Fall 2000) changed the emission-line fluxes by $<5 \%$ in most cases.

Subtracting the best-fitting stellar continuum from the data resulted in a pure emission-line spectrum in which the Balmer and metal (forbidden) lines were optimally corrected for stellar absorption. To measure the integrated emission-line fluxes, we simultaneously modeled the first four Balmer lines- $\mathrm{H} \alpha, \mathrm{H} \beta$, $\mathrm{H} \gamma$, and $\mathrm{H} \delta$-and the strong forbidden lines-[O II] $\lambda \lambda$ 3726, 3729, [O III] $\lambda \lambda 4959,5007$, [N II] $\lambda \lambda 6548,6584$, and [S II] $\lambda \lambda 6716,6731$-assuming Gaussian line profiles. We carried out this fitting twice: on the first iteration, we constrained the redshifts and intrinsic velocity widths of all the lines together, and on the second iteration, we relaxed these constraints and used the best-fitting parameters from the first iteration as initial guesses. This second step was necessary because of uncertainties in the wavelength-dependent instrumental resolution and to account for any small $\left(<50 \mathrm{~km} \mathrm{~s}^{-1}\right)$ residual errors in the wavelength solution, particularly toward the edges of the spectra.

For galaxies with spectra from Moustakas \& Kennicutt (2006), we find that our updated fluxes for the $\mathrm{H} \alpha$ and $\mathrm{H} \beta$ emission lines typically agree with the published fluxes to

\footnotetext{
${ }^{20}$ http://www-astro.physics.ox.ac.uk/ mxc/software/\#ppxf

21 http://star-www.herts.ac.uk/ sarzi
} 
Table 1

Summary of Galaxy Properties, Including Aperture Emission-line Fluxes and (Total) Radio-Continuum Flux Densities

\begin{tabular}{|c|c|c|c|c|c|c|c|c|c|c|c|c|}
\hline Name & $\begin{array}{c}d_{L} \\
(\mathrm{Mpc})\end{array}$ & $\begin{array}{c}a \\
\left({ }^{\prime \prime}\right)\end{array}$ & $\begin{array}{c}b \\
\left({ }^{\prime \prime}\right)\end{array}$ & $\begin{array}{l}\text { P.A. } \\
\left({ }^{\circ}\right)\end{array}$ & $m_{g, \text { total }}$ & $m_{g, \text { aper }}$ & $\mathrm{H} \beta \lambda 4861$ & $\begin{array}{l}\mathrm{O}[\mathrm{III}] \lambda 5007 \\
\quad\left(10^{-14} \mathrm{erg}\right.\end{array}$ & $\begin{array}{l}\mathrm{H} \alpha \lambda 6563 \\
\left.\mathrm{~m}^{-2} \mathrm{~s}^{-1}\right)\end{array}$ & [N II] $\lambda 6716$ & $\begin{array}{c}1.4 \mathrm{GHz} \\
(\mathrm{mJy})\end{array}$ & $\begin{array}{c}150 \mathrm{MHz} \\
(\mathrm{mJy})\end{array}$ \\
\hline Arp $256 \mathrm{~N}$ & 110.3 & 40 & 60 & 90 & 14.32 & 14.32 & $12.9 \pm 0.6$ & $10.6 \pm 0.8$ & $44.5 \pm 1.9$ & $12.9 \pm 1.4$ & 4 & 23 \\
\hline Arp $256 \mathrm{~S}$ & 109.4 & 40 & 40 & 90 & 14.36 & 14.36 & $15.4 \pm 0.6$ & $14.0 \pm 0.5$ & $68.8 \pm 1.4$ & $23.1 \pm 0.9$ & 42 & 158 \\
\hline NGC 0337 & 18.0 & 95 & 55 & 70 & 11.48 & 11.98 & $73.4 \pm 1.7$ & $101.1 \pm 1.5$ & $261 \pm 3$ & $48.2 \pm 2.0$ & 106 & $404^{\mathrm{b}}$ \\
\hline CGCG 436-030 & 125.1 & 35 & 40 & 90 & 14.58 & 14.58 & $6.6 \pm 0.5$ & $5.7 \pm 0.5$ & $32.1 \pm 1.1$ & $13.6 \pm 1.0$ & 50 & 87 \\
\hline NGC 0520 & 30.5 & 140 & 100 & 90 & 11.98 & 11.98 & $14.9 \pm 2.8$ & $12.0 \pm 2.3$ & $41.0 \pm 6.5$ & $26.8 \pm 4.5$ & 176 & $433^{\mathrm{b}}$ \\
\hline
\end{tabular}

Notes.

a $150 \mathrm{MHz}$ flux density from 6C or 7C (Baldwin et al. 1985; Hales et al. 1988, 1990, 1991, 1993a, 1993b, 2007).

b $150 \mathrm{MHz}$ flux density from GLEAM (Wayth et al. 2015; Hurley-Walker et al. 2017).

(This table is available in its entirety in machine-readable form.)

within $10 \%$. For galaxies with spectra from Moustakas et al. (2010), the $\mathrm{H} \alpha$ emission-line fluxes are systematically lower by $\approx 20 \%$, and the $\mathrm{H} \beta$ fluxes are higher by $\approx 10 \%$ relative to the previously published values. We attribute these non-negligible differences to an interpolation error in the spectra analyzed by Moustakas et al. (2010). Finally, as noted in Section 2, spectrophotometry was renormalized by a factor determined by dividing SDSS $g$-band aperture photon fluxes with $g$-band photon fluxes synthesized from the spectra, which typically increased emission-line fluxes by $\approx 10 \%$.

As the revisions to the $\mathrm{H} \alpha$ and $\mathrm{H} \beta$ emission-lines fluxes were not negligible, we ran a series of cross-checks to verify their accuracy. Visual inspection of the plots was used to verify the accuracy of the stellar continuum subtracted for each galaxy. Several diagnostic plots, including BPT and emissionline ratio versus luminosity diagrams, had less scatter when revised emission-line fluxes replaced published emission-line fluxes. Finally, we cross-checked the emission-line fluxes against a simple model where the continuum was assumed to be constant near the relevant emission line and found agreement to within $10 \%$ for high equivalent width lines. Finally, the increase in emission-line fluxes resulting from renormalizing the spectra with SDSS $g$-band photometry is consistent with the offsets measured by Kennicutt et al. (2008) when comparing Moustakas \& Kennicutt (2006) spectra to narrow-band imaging.

\section{SFR Indicator Calibration Sample}

Our SFR calibrations are anchored to Balmer-decrementcorrected $\mathrm{H} \alpha$ luminosities, so we excluded galaxies from the SFR calibration sample if the $\mathrm{H} \alpha$ or $\mathrm{H} \beta$ emission-line fluxes had a signal-to-noise ratio of less than five. The sample size does not strongly depend on the somewhat arbitrary choice of signal-to-noise ratio (many of the galaxies rejected by this threshold are passive ellipticals), but below this threshold, $\mathrm{H} \alpha$-to-H $\beta$ flux ratios often have uncertainties greater than one, resulting in highly uncertain Balmer decrement corrections. Our signal-to-noise threshold for $\mathrm{H} \alpha$ and $\mathrm{H} \beta$ reduced the sample from 161 galaxies to 109 galaxies, which are listed in Table 1.

The Brown et al. (2014b) sample includes LINERS and AGNs where the $\mathrm{H} \alpha$ emission is not the result of star formation. As we illustrate in Figure 2, we excluded these galaxies from the SFR indicator calibration sample using the BPT diagram (Baldwin et al. 1981) and the criterion of Kauffmann et al. (2003). We also considered excluding AGNs

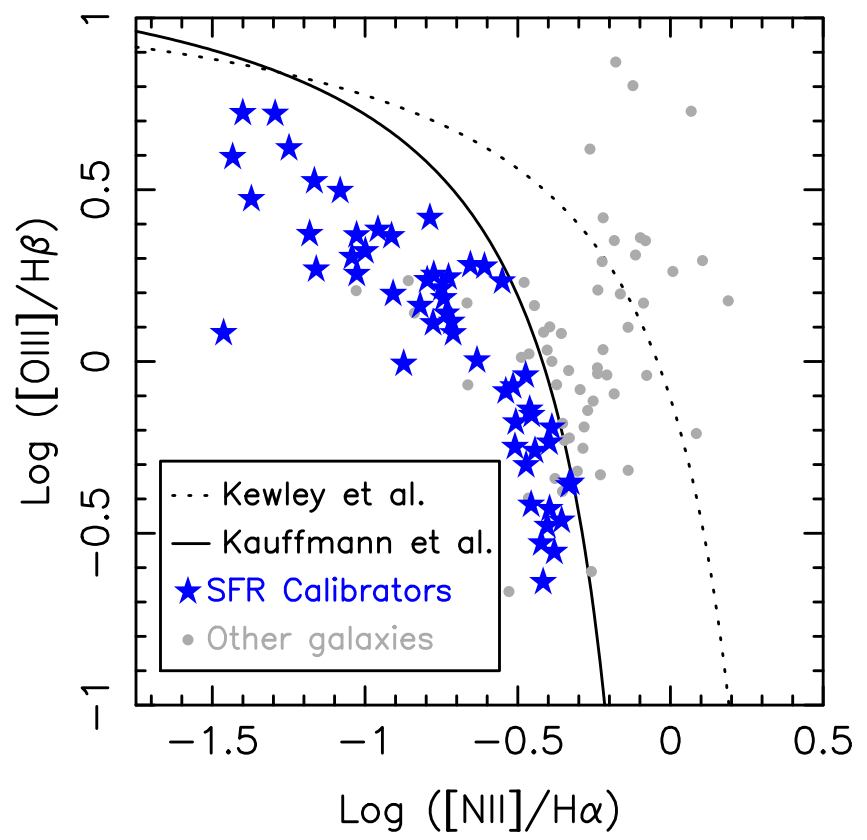

Figure 2. BPT diagram for galaxies in the Brown et al. (2014b) sample. The spectral classification criteria of Kewley et al. (2001) and Kauffmann et al. (2003) are also plotted, and these were used to classify galaxies as star-forming galaxies, AGNs, and potential composite objects. Blue stars show galaxies in the SFR calibration sample, while gray dots denote other galaxies, including those with low signal-to-noise emission-line measurements.

identified using the mid-infrared color criterion of Stern et al. (2005), but this criterion also excludes some low-metallicity dwarf galaxies that we wish to keep in the sample. Finally, as we wanted our SEDs to be representative of entire galaxies, we excluded galaxies from the SFR calibration sample if the $g$-band aperture and total magnitudes differed by more than 0.75 mag. Thus, by construction, we expect that our relations derived from entire galaxies will differ from those using subregions of galaxies and $\mathrm{H}$ II regions (e.g., Calzetti et al. 2007; Relaño et al. 2007; Kennicutt et al. 2009). Our criteria reduced our final SFR indicator calibration sample to 66 galaxies, although for any given calibration, fewer galaxies are used due to data coverage and signal-to-noise limitations.

The optical color-magnitude diagram of the Brown et al. (2014b) sample and the SFR indicator calibration sample are provided in Figure 3. The SFR indicator calibration sample spans $-24<M_{r}<-12$ and $-0.3<u-r<2.3$, and includes galaxies with optical colors approaching those of 


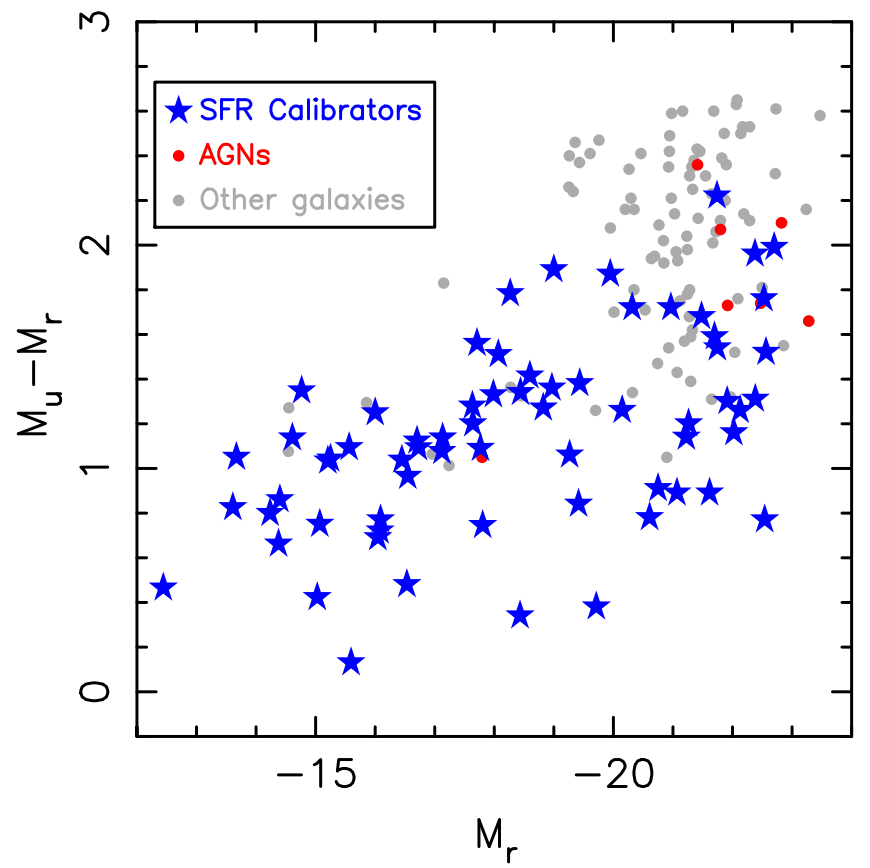

Figure 3. SDSS optical color-magnitude diagram for the sample. Galaxies in the SFR indicator calibration sample are shown with blue stars, BPT-selected AGNs are denoted by red circles, and other galaxies are shown in gray (including galaxies with low signal-to-noise emission-line fluxes). The SFR indicator calibration galaxies span a broad range of optical color and absolute magnitude.

passive galaxies. This broad distribution of optical properties reflects the deliberate targeting of galaxies spanning a broad range of optical properties by Moustakas \& Kennicutt (2006) and Moustakas et al. (2010).

We plot the mid-infrared color-magnitude diagrams of the sample in Figure 4, and this figure provides several reasons for caution when using SFR indicators. Unlike the optical colormagnitude diagram, there is a significant gap between the SFR indicator calibration sample and passive galaxies. Several of the galaxies that fall between the star-forming and passive loci are forming stars, but their spectra do not meet the criteria for inclusion in the SFR calibration sample. For example, NGC 3190 and NGC 4725 both lack detectable $\mathrm{H} \beta$ emission in their drift-scan spectra, but both show clear evidence for star formation in GALEX images and SINGS continuum-subtracted $\mathrm{H} \alpha$ images (Kennicutt et al. 2003). Our SFR indicator calibration sample does not probe the lowest specific star formation rates (sSFRs), and this may be true of other calibrations in the literature that have similar limitations.

At fixed stellar mass, one may expect different SFR indicators to have comparable logarithmic luminosity ranges, but this is not the case for the WISE W3 and W4 bands. Figure 4 illustrates that the distributions of the $W 3$ and $W 4$ luminosities at fixed $W 2$ absolute magnitude (or approximate stellar mass) differ considerably from each other. When we fit to the midinfrared color-magnitude relations for the SFR calibration sample, we find that both relations are tilted and the data show significant scatter about these relations, which is to be expected as mid-infrared luminosity is not a linear function of SFR (e.g., Lee et al. 2013; Catalán-Torrecilla et al. 2015); the sSFR would not necessarily be constant with stellar mass; and the starforming "main sequence" has significant scatter at fixed mass. The $1 \sigma$ scatter for the $M_{W 2}-M_{W 3}$ colors about the best-fit relation is $\sim 0.6 \mathrm{mag}$, which is considerably less than the $1 \sigma$ scatter for the $M_{W 2}-M_{W 4}$ color data, which is $\sim 1 \mathrm{mag}$. As the sSFRs derived from the $\mathrm{H} \alpha$ luminosities span approximately an order of magnitude, the relatively narrow range of $M_{W 2}-M_{W 3}$ colors may imply that WISE W3 has a limited dynamic range as an SFR indicator. Furthermore, galaxies in the SFR calibration sample have colors that span $0.0<M_{W 3}-M_{W 4}<2.3$, so in many instances SFRs determined with the WISE W3 and W4 bands will differ significantly from each other.

\section{Star Formation Rate Indicator Calibrations}

Our SFR indicator calibrations are anchored to Balmerdecrement-corrected $\mathrm{H} \alpha$ luminosities assuming a Fitzpatrick (1999) dust attenuation curve with $R_{V}=3.1$ and Case B recombination with an effective temperature of $10,000 \mathrm{~K}$ and $n_{e}=10^{2} \mathrm{~cm}^{-3}$, where the ratio of $\mathrm{H} \alpha$ luminosity to $\mathrm{H} \beta$ luminosity is 2.86 (Storey \& Hummer 1995; Dopita \& Sutherland 2003). This choice is transparent and easier to replicate than more complex modeling of galaxy SEDs and dust geometry, but its simplifying assumptions must be wrong in detail (e.g., obscuration by a dusty screen).

The assumptions we used when determining Balmerdecrement-corrected $\mathrm{H} \alpha$ luminosities probably have limited impact on SFR calibrations, and this is discussed in detail by Kennicutt et al. (2009). For example, Calzetti et al. (2007) found that the attenuations for $\mathrm{H} \alpha$ determined using the Balmer decrement technique show no systematic offset relative to those determined with $\mathrm{Pa} \alpha / \mathrm{H} \alpha$ ratios. Furthermore, when we fitted models to the relationship between the SFR indicator luminosity and Balmer-decrement-corrected $\mathrm{H} \alpha$ luminosity, we found that the parameter values changed by $\lesssim 2 \sigma$ when we substituted a Calzetti et al. (2000) dust attenuation law for our default Fitzpatrick (1999) dust attenuation law.

In Figure 5, we plot the ratio of the $\mathrm{H} \alpha$ to $\mathrm{H} \beta$ flux as a function of $\mathrm{H} \alpha$ luminosity, along with the expected ratio for the $10,000 \mathrm{~K}$ Case $\mathrm{B}$ recombination. The value of the $\mathrm{H} \alpha$ luminosity divided by the $\mathrm{H} \beta$ luminosity for Case $\mathrm{B}$ recombination can vary from 2.75 to 3.04 for temperatures ranging from $20,000 \mathrm{~K}$ to $5000 \mathrm{~K}$, but we do not expect this source of error to dominate the observed scatter in the SFR indicator calibrations. As has been reported in previous literature (e.g., Lee et al. 2009), blue compact dwarf galaxies that have low $\mathrm{H} \alpha$ luminosities (but high sSFRs) also have relatively little dust obscuration, and the $\mathrm{H} \alpha$ to $\mathrm{H} \beta$ flux ratios asymptote toward the expected range for Case B recombination.

Figure 6 shows the sSFRs of the sample galaxies as a function of their stellar mass. SFRs were determined using

$$
\operatorname{SFR}\left(M_{\odot} \mathrm{yr}^{-1}\right)=5.5 \times 10^{-42} L_{\mathrm{H} \alpha}\left(\mathrm{erg} \mathrm{s}^{-1}\right)
$$

(Kennicutt et al. 2009), which uses a Kroupa (2001) IMF and a constant SFR. Approximate stellar masses were determined using WISE $W 1$ and $W 2$ photometry and the relation of Cluver et al. (2014), with the addition of 0.07 dex to convert from a Chabrier (2003) IMF to a Kroupa (2001) IMF. The sSFRs decrease with increasing stellar mass, and at fixed stellar mass the sSFRs have a range of two orders of magnitude. The "star-forming main sequence" (e.g., Noeske et al. 2007; Elbaz et al. 2011) is not particularly evident in Figure 6, which is an artifact of the sample selection that emphasized spanning the parameter space rather than providing a flux-limited galaxy sample (Moustakas \& Kennicutt 2006; Moustakas et al. 2010). 

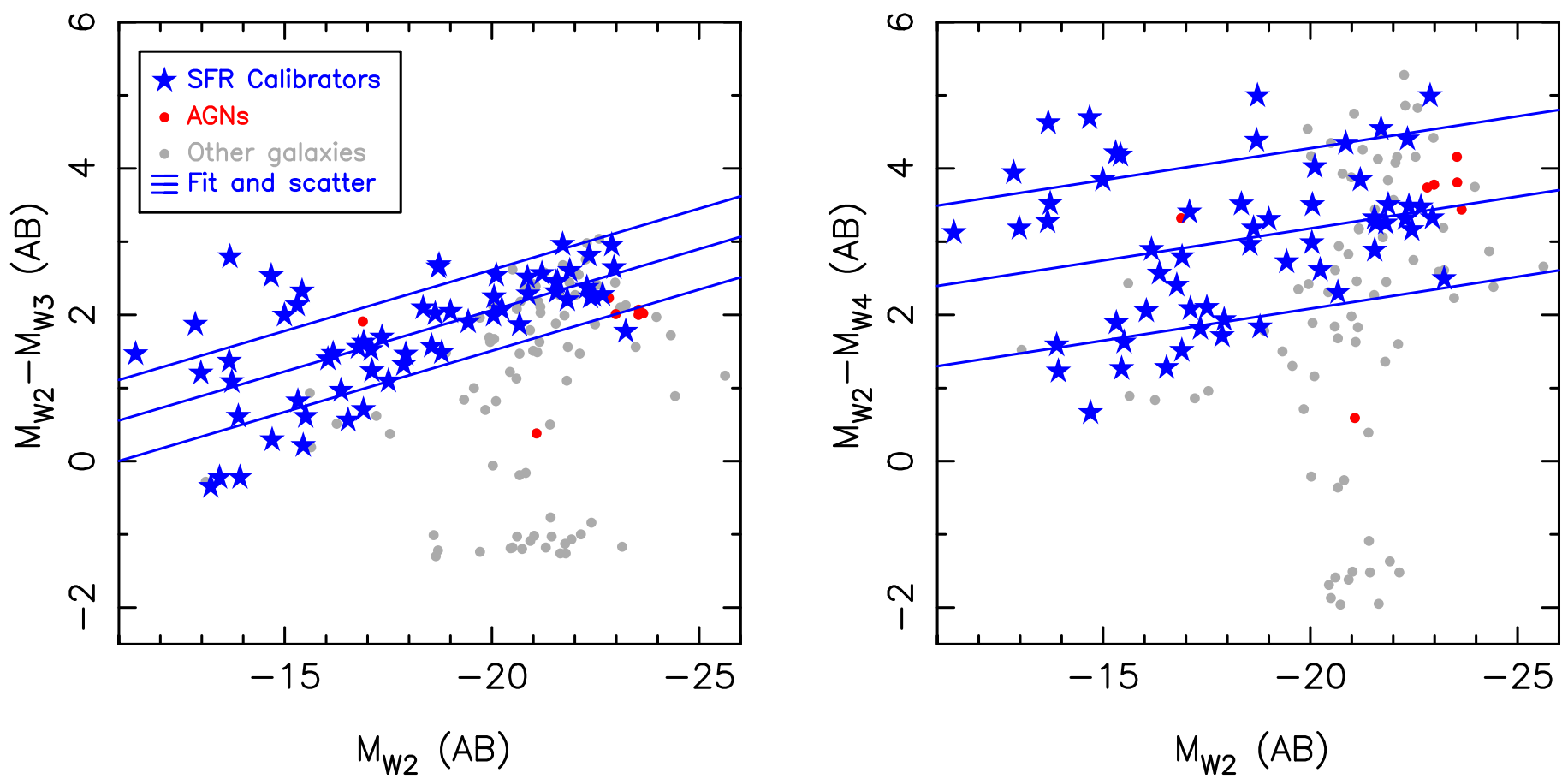

Figure 4. WISE mid-infrared color-magnitude diagrams for the sample. Compared to the optical color-magnitude diagram, SFR indicator calibration galaxies are clearly separated from the locus of passive galaxies (located at the bottom right of both panels). While both WISE W3 and W4 luminosities are used as SFR indicators, the widths of the $M_{W 2}-M_{W 3}$ and $M_{W 2}-M_{W 4}$ distributions differ considerably from each other, and this may imply that $W 3$ has a limited dynamic range as an SFR indicator.
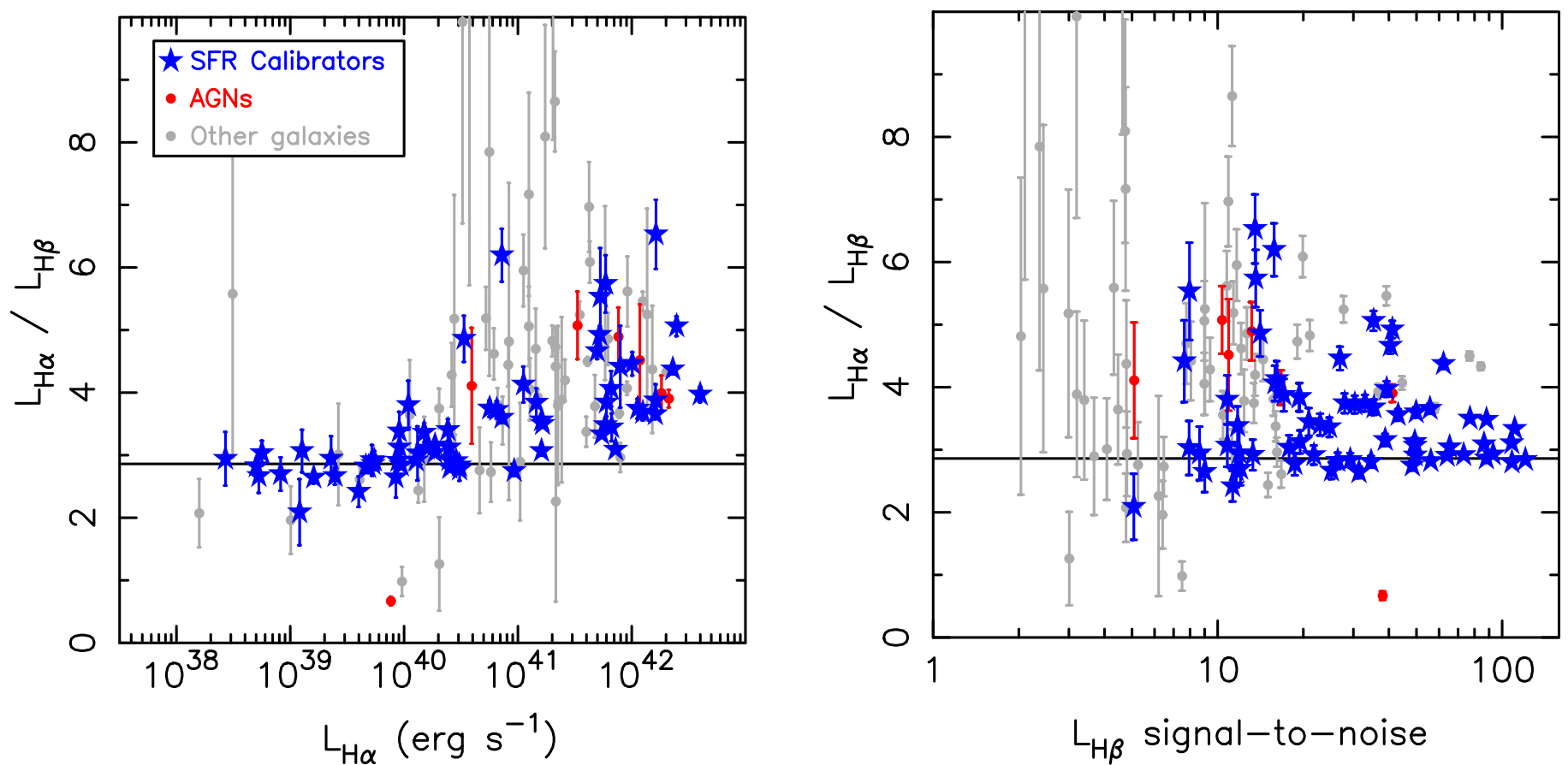

Figure 5. Ratio of observed $\mathrm{H} \alpha$ luminosity to observed $\mathrm{H} \beta$ luminosity, as a function of $\mathrm{H} \alpha$ luminosity (left panel) and $\mathrm{H} \beta$ signal-to-noise ratio (right panel). Galaxies used for the star formation rate calibration are shown with blue stars, BPT-selected AGNs are shown with red dots, and other galaxies (including those with low signalto-noise emission-line fluxes) are shown in gray. Dust obscuration increases with increasing luminosity, while at low luminosities the ratio of $\mathrm{H} \alpha$ luminosity to $\mathrm{H} \beta$ luminosity approaches the value expected for Case B recombination. The spread of the $\mathrm{H} \alpha$ luminosity to $\mathrm{H} \beta$ luminosity ratios does depend on the signal-to-noise ratio, with spuriously low values being associated with mediocre signal-to-noise ratios.

For consistency with (much of) the previous literature, we use powers in units of $\mathrm{W} \mathrm{Hz}^{-1}$ for the radio continuum and $\nu L_{\nu}$ in units of erg $\mathrm{s}^{-1}$ for the ultraviolet and mid-infrared, where the frequency $\nu$ is determined from the effective wavelength of the relevant filter. In the ultraviolet and mid-infrared, the flux density is given by

$$
f_{\nu}=3631 \mathrm{Jy} \times 10^{-0.4 m}
$$

where $m$ is the $\mathrm{AB}$ apparent magnitude. We caution that some flux densities presented in the literature do not use this 


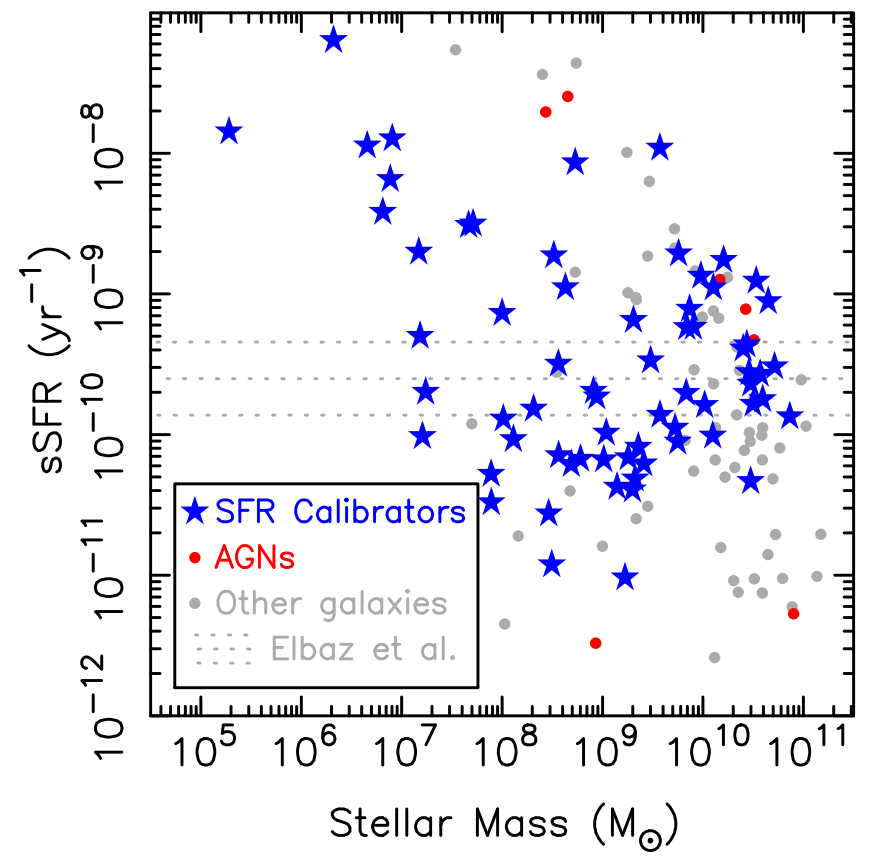

Figure 6. sSFR as a function of galaxy mass. sSFRs decrease with increasing stellar mass, and at fixed stellar mass the sSFRs have a range of two orders of magnitude. The location of the "star-forming main sequence" is illustrated with the 16th, 50th, and 84th percentiles from Elbaz et al. (2011). The "star-forming main sequence" is not particularly evident in our sample, which is an artifact of the sample selection that emphasized spanning the parameter space (Moustakas \& Kennicutt 2006; Moustakas et al. 2010)

definition, and this can result in systematic offsets of several percent. The effective wavelengths of the relevant filters are presented in Table 2. The effective wavelength depends on the weighting function used, corresponding to the assumed spectrum of the source being observed, so we choose to use effective wavelengths as published by the relevant survey/ satellite teams. For the calibration of the radio continuum as an SFR indicator, we used the flux densities from NVSS and TGSS ADR1 (Condon et al. 1998, 2002; Intema et al. 2017), and frequencies of $1.40 \mathrm{GHz}$ or $150 \mathrm{MHz}$.

To model the relationship between the SFR indicator luminosity and Balmer-decrement-corrected $\mathrm{H} \alpha$ luminosity, we used two parameterizations. The first is a power law where the index and normalization are free parameters, which is commonly used and thus simplifies direct comparisons with the previous literature. Table 3 provides an incomplete list of power-law SFR calibrations from previous literature, including models with power-law indices fixed to one (e.g., Kennicutt et al. 2009). Table 3 provides at least four calibrations for each filter, with an emphasis on calibrations based on $\mathrm{H} \alpha$ and $\mathrm{Pa} \alpha$, which aids direct comparison with our work. ${ }^{22}$ To simplify comparisons of different models, we rewrote the parameterizations from the previous literature so that they are a function of $\mathrm{H} \alpha$ luminosity with the normalization being the SFR indicator luminosity of a galaxy with an $\mathrm{H} \alpha$ luminosity of $10^{40} \mathrm{erg} \mathrm{s}^{-1}$.

The power-law parameterization assumes two galaxies with the same SFR but very different masses and metallicities will

\footnotetext{
${ }^{22}$ Please note that Table 3 does not include some calibrations that utilize the total infrared luminosity (e.g., Goto et al. 2011; Rujopakarn et al. 2013), and some papers listed in Table 3 use several different calibration methods (e.g., Rieke et al. 2009; Davies et al. 2016).
}

Table 2

Ultraviolet and Mid-infrared Filter Effective Wavelengths

\begin{tabular}{lcl}
\hline \hline Filter & Effective Wavelength & Reference \\
\hline GALEX FUV & $1538.6 \AA$ & Morrissey et al. (2007) \\
GALEX NUV & $2315.7 \AA$ & Morrissey et al. (2007) \\
IRAC $3.6 \mu \mathrm{m}$ & $3.55 \mu \mathrm{m}$ & Fazio et al. (2004) \\
IRAC $4.5 \mu \mathrm{m}$ & $4.439 \mu \mathrm{m}$ & Fazio et al. (2004) \\
IRAC $5.8 \mu \mathrm{m}$ & $5.731 \mu \mathrm{m}$ & Fazio et al. (2004) \\
IRAC $8.0 \mu \mathrm{m}$ & $7.872 \mu \mathrm{m}$ & Fazio et al. (2004) \\
MIPS $24 \mu \mathrm{m}$ & $23.675 \mu \mathrm{m}$ & Engelbracht et al. (2007) \\
WISE $W 1$ & $3.3526 \mu \mathrm{m}$ & Jarrett et al. (2011) \\
WISE $W 2$ & $4.6028 \mu \mathrm{m}$ & Jarrett et al. (2011) \\
WISE $W 3$ & $11.5608 \mu \mathrm{m}$ & Jarrett et al. (2011) \\
WISE $W 4$ & $22.8 \mu \mathrm{m}$ & Brown et al. (2014a) \\
\hline
\end{tabular}

have the same SFR indicator luminosity, which may not necessarily be the case. For example, we might expect that a metal-rich $L^{*}$ galaxy will have higher dust content and higher mid-infrared luminosity at a given SFR than a metal-poor dwarf galaxy with the same SFR. This motivated our second parameterization of the relationship between the SFR indicator luminosity and SFR.

Our second parameterization assumes that the SFR indicator luminosity is directly proportional to the SFR for galaxies of a given mass, with the normalization being a power-law function of galaxy mass. To simplify the use of this parameterization, we used Spitzer $4.5 \mu \mathrm{m}$ and WISE W2 luminosities as stellar mass proxies. ${ }^{23}$ This parameterization has the same number of free parameters as the power-law models, but may be less prone to error when extrapolated to high and low SFRs if its underlying assumption is valid (i.e., luminosity is a linear function of SFR for galaxies of a given mass).

For each relation, the $1 \sigma$ scatter of the data about the best fit was determined by finding the scatter that encompassed $68 \%$ of the data, and any galaxies more than $2 \sigma$ from the best-fit relation were flagged as potential outliers. Wide-field surveys cannot always apply stringent BPT criteria, so we also present measurements of the scatter using galaxies that meet the less stringent BPT criterion of Kewley et al. (2001). This second measurement of the scatter may overestimate the scatter for magnitude-limited samples, as AGNs and LIRGs are overrepresented in the Brown et al. (2014b) sample. Parameter values are presented for galaxies with $\mathrm{H} \alpha$ luminosities of $10^{40} \mathrm{erg} \mathrm{s}^{-1}$ (rather than extrapolating to $1 \mathrm{erg} \mathrm{s}^{-1}$ ) to reduce quoted uncertainties.

As a sobriety test for the relations presented in this paper, in Figure 7 we present $W 2(4.6 \mu \mathrm{m})$ luminosity as a function of Balmer-decrement-corrected $\mathrm{H} \alpha$ luminosity. Although WISE $W 2$ is usually a proxy for stellar mass rather than for SFR, nearinfrared luminosity does depend on stellar population age (e.g., Bruzual \& Charlot 2003) and it thus is not entirely independent of SFR. The power-law fit to the WISE W2 data has an index close to one, and the scatter around the best-fit power law is $0.4 \mathrm{dex}$, which is smaller than the scatter seen in SSFR versus stellar mass for our sample (illustrated by Figure 6). Galaxies with lower sSFRs than the BPT-selected calibration sample fall to the left of the power-law fit, having significant WISE W2

\footnotetext{
23 Although the Spitzer $4.5 \mu \mathrm{m}$ and WISE W2 bands include $\operatorname{Br} \alpha$, for most star-forming galaxies, the Br $\alpha$ emission-line fluxes (e.g., Imanishi et al. 2010) are small compared to the Spitzer and WISE broadband fluxes.
} 
Table 3

A Selection of Star Formation Rate Indicator Calibrations from Previous Literature

\begin{tabular}{|c|c|c|}
\hline Indicator $^{\mathrm{a}}$ & $\mathrm{Fit}^{\mathrm{b}}$ & References \\
\hline $\log L_{\mathrm{FUV}}{ }^{\mathrm{c}}$ & $42.03+0.74 \times\left(\log L_{\mathrm{H} \alpha, \text { Corr }}-40\right)$ & Lee et al. (2009) \\
\hline $\log L_{\mathrm{FUV}}{ }^{\mathrm{c}}$ & $42.09+\left(\log L_{\mathrm{H} \alpha, \text { Corr }}-40\right)$ & Hao et al. (2011) \\
\hline $\log L_{\mathrm{FUV}}{ }^{\mathrm{c}}$ & $42.87+0.74 \times\left(\log L_{\mathrm{H} \alpha, \text { Corr }}-40\right)$ & Davies et al. (2016) \\
\hline $\log L_{\mathrm{FUV}}{ }^{\mathrm{c}}$ & $41.70+1.11 \times\left(\log L_{\mathrm{H} \alpha, \text { Corr }}-40\right)$ & Jaiswal \& Omar (2016) \\
\hline $\log L_{8} \mu \mathrm{m}$ & $41.80+0.92 \times\left(\log L_{\mathrm{H} \alpha, \text { Corr }}-40\right)$ & Wu et al. (2005) \\
\hline $\log L_{8} \mu \mathrm{m}$ & $41.56+0.94 \times\left(\log L_{\mathrm{H} \alpha, \text { Corr }}-40\right)$ & Calzetti et al. $(2007)^{\mathrm{d}, \mathrm{e}}$ \\
\hline $\log L_{8} \mu \mathrm{m}$ & $41.97+1.14 \times\left(\log L_{\mathrm{H} \alpha, \text { Corr }}-40\right)$ & Zhu et al. (2008) \\
\hline $\log L_{8} \mu \mathrm{m}$ & $41.67+\left(\log L_{\mathrm{H} \alpha, \text { Corr }}-40\right)$ & Kennicutt et al. (2009) \\
\hline $\log L_{W 3}$ & $41.61+\left(\log L_{\mathrm{H} \alpha, \text { Corr }}-40\right)$ & Jarrett et al. (2013) \\
\hline $\log L_{W 3}$ & $41.27+0.97 \times\left(\log L_{\mathrm{H} \alpha, \text { Corr }}-40\right)$ & Lee et al. (2013) \\
\hline $\log L_{W 3}$ & $41.29+0.88 \times\left(\log L_{\mathrm{H} \alpha, \text { Corr }}-40\right)$ & Cluver et al. (2014) \\
\hline $\log L_{W 3}$ & $41.67+0.83 \times\left(\log L_{\mathrm{H} \alpha, \text { Corr }}-40\right)$ & Davies et al. (2016) \\
\hline $\log L_{W 4}$ & $41.43+\left(\log L_{\mathrm{H} \alpha, \text { Corr }}-40\right)$ & Jarrett et al. (2013) \\
\hline $\log L_{W 4}$ & $41.15+1.04 \times\left(\log L_{\mathrm{H} \alpha, \text { Corr }}-40\right)$ & Lee et al. (2013) \\
\hline $\log L_{W 4}$ & $40.61+1.22 \times\left(\log L_{\mathrm{H} \alpha, \text { Corr }}-40\right)$ & Cluver et al. (2014) \\
\hline $\log L_{W 4}$ & $41.26+\left(\log L_{\mathrm{H} \alpha, \text { Corr }}-40\right)$ & Catalán-Torrecilla et al. (2015) \\
\hline $\log L_{W 4}$ & $40.84+1.36 \times\left(\log L_{\mathrm{H} \alpha, \text { Corr }}-40\right)$ & Catalán-Torrecilla et al. (2015) \\
\hline $\log L_{W 4}$ & $41.33+1.20 \times\left(\log L_{\mathrm{H} \alpha, \text { Corr }}-40\right)$ & Davies et al. (2016) \\
\hline $\log L_{24 \mu \mathrm{m}}$ & $41.11+1.12 \times\left(\log L_{\mathrm{H} \alpha, \text { Corr }}-40\right)$ & Wu et al. (2005) \\
\hline $\log L_{24 \mu \mathrm{m}}$ & $41.13+1.13 \times\left(\log L_{\mathrm{H} \alpha, \text { Corr }}-40\right)$ & Calzetti et al. (2007) \\
\hline $\log L_{24 \mu \mathrm{m}}$ & $41.12+1.21 \times\left(\log L_{\mathrm{H} \alpha, \text { Corr }}-40\right)$ & Relaño et al. (2007) \\
\hline $\log L_{24 \mu \mathrm{m}}$ & $41.10+1.18 \times\left(\log L_{\mathrm{H} \alpha, \text { Corr }}-40\right)$ & Zhu et al. (2008) \\
\hline $\log L_{24 \mu \mathrm{m}}$ & $41.33+\left(\log L_{\mathrm{H} \alpha, \text { Corr }}-40\right)$ & Kennicutt et al. (2009) \\
\hline $\log L_{24 \mu \mathrm{m}}$ & $41.53+1.18 \times\left(\log L_{\mathrm{H} \alpha, \text { Corr }}-40\right)$ & Rieke et al. (2009) ${ }^{\mathrm{e}}$ \\
\hline $\log P_{1.4 \mathrm{GHz}}$ & $20.20+\left(\log L_{\mathrm{H} \alpha, \text { Corr }}-40\right)$ & Condon (1992) \\
\hline $\log P_{1.4 \mathrm{GHz}}$ & $20.16+\log \left(L_{\mathrm{H} \alpha, \text { Corr }}-40\right)$ when $\log P_{1.4 \mathrm{GHz}}>21.81$ & Bell (2003) \\
\hline $\log P_{1.4 \mathrm{GHz}}$ & $20.05+\log \left(L_{\mathrm{H} \alpha, \text { Corr }}-40\right)$ & Kennicutt et al. (2009) \\
\hline $\log P_{1.4 \mathrm{GHz}}$ & $19.62+1.18 \times\left(\log L_{\mathrm{H} \alpha, \text { Corr }}-40\right)$ & Boselli et al. (2015) \\
\hline
\end{tabular}

Notes.

${ }^{\mathrm{a}} \mathrm{UV}$ and mid-infrared luminosities are presented in units of $\mathrm{erg} \mathrm{s}^{-1}$ while radio powers are presented in units of $\mathrm{W} \mathrm{Hz}{ }^{-1}$.

${ }^{\mathrm{b}}$ In some instances, we converted SFRs to $L_{\mathrm{H} \alpha, \text { Corr }}$ using $\operatorname{SFR}\left(M_{\odot} \mathrm{yr}^{-1}\right)=7.9 \times 10^{-42} L_{\mathrm{H} \alpha}\left(\mathrm{erg} \mathrm{s}^{-1}\right)$ for a $\operatorname{Salpeter}(1955) \operatorname{IMF}, \operatorname{SFR}\left(M_{\odot} \mathrm{yr}^{-1}\right)=5.5 \times$ $10^{-42} L_{\mathrm{H} \alpha}\left(\mathrm{erg} \mathrm{s}^{-1}\right)$ for a Kroupa (2001) IMF, $\operatorname{SFR}\left(M_{\odot} \mathrm{yr}^{-1}\right)=1.2 \times 10^{-41} L_{\mathrm{H} \alpha}\left(\mathrm{erg} \mathrm{s}^{-1}\right)$ for a Chabrier $(2003) \operatorname{IMF}$, and $\operatorname{SFR}\left(M_{\odot} \mathrm{yr}^{-1}\right)=5.1 \times$ $10^{-42} L_{\mathrm{H} \alpha}\left(\mathrm{erg} \mathrm{s}^{-1}\right)$ for a Baldry \& Glazebrook (2003) IMF.

${ }^{c}$ GALEX FUV luminosities have been corrected for dust extinction, and we refer readers to the original papers for the relevant details.

${ }^{\mathrm{d}}$ The Calzetti et al. (2007) $8 \mu \mathrm{m}$ relation is for luminosity per $\mathrm{kpc}^{2}$.

${ }^{\mathrm{e}}$ We adopt $L_{\mathrm{Pa} \alpha}=0.128 L_{\mathrm{H} \alpha}$ (Hummer \& Storey 1987).

emission but low SFRs. We remind adventurous readers to not use WISE W2 as an SFR indicator.

\subsection{Ultraviolet}

To use FUV as an SFR indicator, one must model the dust extinction and the intrinsic SED of the galaxy stellar population. Although one can model entire SEDs to derive stellar populations and dust extinction (e.g., da Cunha et al. 2008; Noll et al. 2009), this is not always practical for wide-field surveys (e.g., much of the southern sky currently lacks ugriz imaging while 2MASS $J H K_{S}$ imaging is shallow). As NUV imaging is almost always available with FUV imaging, we adopted corrections for dust-extinction corrections that are a function of $M_{\mathrm{FUV}}-M_{\mathrm{NUV}}$ color. This effectively makes our FUV calibrations composites with NUV, whereas monochromatic calibrations are available for all of the other bands presented in this paper.

In Figure 8, we present two FUV calibrations that use different stellar population and dust-extinction corrections. In the left panel of Figure 8, we assumed that the stellar population spectrum of star-forming galaxies has a dust-free color of $M_{\mathrm{FUV}}-M_{\mathrm{NUV}}=0$, which is comparable to the bluest galaxies in our sample and young populations (e.g., Gil de Paz et al. 2007; Lisker \& Han 2008), and then corrected for internal dust extinction using a Calzetti et al. (2000) extinction law. In the right panel of Figure 8, we assumed that the stellar population spectrum of star-forming galaxies has a dust-free color of $M_{\mathrm{FUV}}-M_{\mathrm{NUV}}=0.022$ (Hao et al. 2011), and we used the empirical model of FUV dust attenuation as a function of $M_{\mathrm{FUV}}-M_{\mathrm{NUV}}$ from Hao et al. (2011). Both dust corrections 


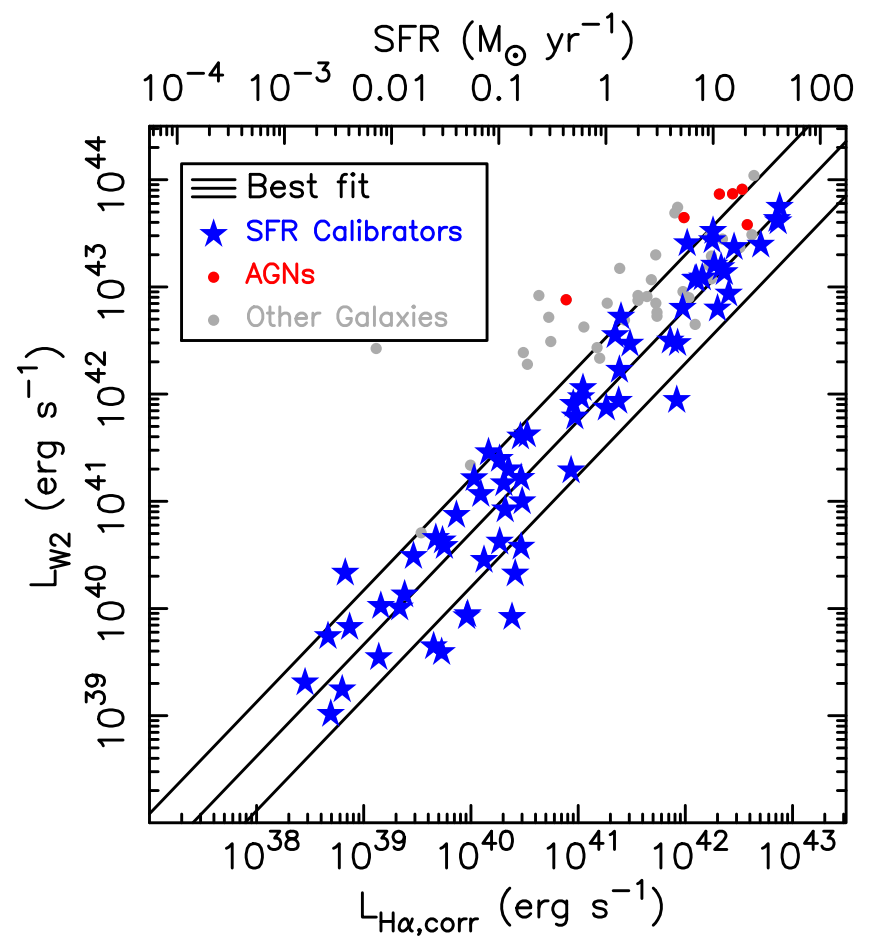

Figure 7. WISE $W 2(4.6 \mu \mathrm{m})$ luminosity as a function of Balmer-decrementcorrected $\mathrm{H} \alpha$ luminosity. A power-law fit to the data, and the $\pm 1 \sigma$ scatter of the data, is shown with black lines. As $W 2$ is a better tracer of stellar mass than SFR, this plot illustrates the luminosity-luminosity correlations in the sample. Unlike fits to data at longer wavelengths, the best-fit power law has an index close to one, while the scatter of the data around the fit is relatively large $(0.4 \operatorname{dex})$.

make assumptions about stellar populations and dust obscuration that must be wrong for many individual star-forming galaxies, but (as we discuss below) the impact of these assumptions is reduced via the empirical calibration of FUV with $\mathrm{H} \alpha$.

In Figure 8, we present the dust-corrected GALEX FUV luminosity as a function of the Balmer-decrement-corrected $\mathrm{H} \alpha$ luminosity. Power-law fits to the data are also plotted in Figure 8 , and the relevant parameter values provided in Table 4. Both fits have power-law indices within $10 \%$ of the expected value of one, and the fits are comparable to the predicted relationship between FUV and $\mathrm{H} \alpha$ from STARBURST99 (Leitherer et al. 1999) for a 100 Myr old stellar population with a Kroupa IMF (Hao et al. 2011). As the powerlaw fits have indices close to one, we have not attempted to use our alternative parameterization to calibrate the FUV data. Empirical relations for GALEX FUV luminosity as a function of $\mathrm{H} \alpha$ luminosity (Lee et al. 2009; Davies et al. 2016; Jaiswal \& Omar 2016) show significant offsets with respect to each other and our work, and this may be partially explained by the different models for correcting dust attenuation. Unfortunately, the scatter of the data around our best-fit power laws is $\sim 0.3$ dex, and thus not much better than what was achieved with WISE W2.

\subsection{Mid-infrared}

Mid-infrared emission from star-forming galaxies is dominated by the blackbody radiation from warm dust and emission features attributed to PAHs, and thus mid-infrared emission resulting from star formation has dependencies on dust content (and thus metallicity), geometry, and temperature. Furthermore, the mid-infrared emission from galaxies can include contributions from dust heated by old stellar populations ("galactic cirrus"), AGNs, and the Rayleigh-Jeans tail of stellar spectra. Mid-infrared emission from galaxies is thus the result of complex astrophysics, and it is a fortunate accident that the relationship between star formation and mid-infrared luminosity can be empirically modeled with relatively simple functions (e.g., Wu et al. 2005; Kennicutt et al. 2009; Catalán-Torrecilla et al. 2015).

We present the relationship between mid-infrared luminosity and Balmer-decrement-corrected $\mathrm{H} \alpha$ luminosity in Figures 912. We did not subtract the stellar continuum from the midinfrared luminosities (i.e., to produce a "dust" luminosity), as tests with the stellar continuum subtracted did not reduce the scatter and changed the fit parameter values by $2 \sigma$ or less. Figures 9-12 show the Spitzer IRAC $8 \mu \mathrm{m}$, WISE W3 $(12 \mu \mathrm{m})$, WISE W4 $(22.8 \mu \mathrm{m})$, and Spitzer MIPS $24 \mu \mathrm{m}$ bands, respectively. In all of the figures, gray lines denote the power-law fits taken from a subset of previous literature $(\mathrm{Wu}$ et al. 2005; Relaño et al. 2007; Zhu et al. 2008; Kennicutt et al. 2009; Jarrett et al. 2013; Lee et al. 2013; Cluver et al. 2014; Catalán-Torrecilla et al. 2015; Davies et al. 2016).

In Figures 9-12, we provide power-law fits to the data and the relevant parameter values are provided in Table 4 . For all four mid-infrared bands, we find power-law indices consistent with 1.3. Some of the previous studies find or adopt power-law indices of close to unity (i.e., Calzetti et al. 2007; Kennicutt et al. 2009; Jarrett et al. 2013; Lee et al. 2013), and when these fits are extrapolated to low luminosities, they can disagree with our fits by an order of magnitude. However, given that the mid-infrared emission from PAHs and dust depends on temperature and metallicity (e.g., Engelbracht et al. 2005, 2008; Wu et al. 2006; Calzetti et al. 2007; Draine et al. 2007; Smith et al. 2007), there is no expectation that the power-law index for the mid-infrared calibrations for entire galaxies should be one.

Galaxies with $\mathrm{H} \alpha$ luminosities of $10^{40} \mathrm{erg} \mathrm{s}^{-1}$ have midinfrared luminosities of $\sim 10^{40.8} \mathrm{erg} \mathrm{s}^{-1}$ for all four mid-infrared bands. The scatter around the best-fit relations decreases with increasing wavelength, dropping from $0.33 \mathrm{dex}$ for Spitzer IRAC $8 \mu \mathrm{m}$ to 0.18 dex for Spitzer MIPS $24 \mu \mathrm{m}$. The scatter is much larger than the uncertainties from the emission-line measurements, photometry, and distance errors, and we thus conclude that decreasing scatter with increasing wavelength is an intrinsic feature of these relations.

Our fits to the mid-infrared luminosity as a function of Balmer-decrement-corrected $\mathrm{H} \alpha$ luminosity (or SFRs) have steeper power-law indices than those determined (or adopted) in most previous literature (with the exception being CatalánTorrecilla et al. 2015). Apart from when a power-law index of one is adopted (e.g., Kennicutt et al. 2009; Jarrett et al. 2013), the largest discrepancies occur for studies that are limited to relatively high luminosities (i.e., $L_{\mathrm{H} \alpha \text {,Corr }}>10^{40} \mathrm{erg} \mathrm{s}^{-1}$ ). This includes most of the calibrations of Spitzer $8 \mu \mathrm{m}$ and WISE W3 from the prior literature. In contrast, studies that approach our luminosity limits, such as those by Relaño et al. (2007) and Catalán-Torrecilla et al. (2015), have power-law indices that agree with ours to within 0.1 . Furthermore, several previous studies show dwarf galaxies falling below their fits to the data (e.g., Wu et al. 2005; Kennicutt et al. 2009). We thus conclude that differences between our power-law indices and those from the literature are primarily the result of our broad luminosity range, and that extrapolations of some relations from the previous literature can result in underestimates of SFRs. 
Table 4

Star Formation Rate Indicator Calibrations

\begin{tabular}{|c|c|c|c|c|c|}
\hline Indicator $^{\mathrm{a}}$ & Fit & $\begin{array}{c}\sigma_{\mathrm{H} \alpha, \mathrm{BPT}} \\
(\operatorname{dex})\end{array}$ & $\begin{array}{c}\sigma_{\mathrm{H} \alpha, \text { More }}{ }^{\mathrm{b}} \\
(\mathrm{dex})\end{array}$ & $\begin{array}{c}>2 \sigma \\
\text { Fraction }\end{array}$ & $n$ \\
\hline $\log L_{\mathrm{FUV}}+2 \times\left(M_{\mathrm{FUV}}-M_{\mathrm{NUV}}\right)$ & $(42.42 \pm 0.05)+(0.96 \pm 0.03) \times\left(\log L_{\mathrm{H} \alpha, \text { Corr }}-40\right)$ & 0.35 & 0.39 & 0.03 & 62 \\
\hline $\log L_{\mathrm{FUV}}+1.532 \times\left(M_{\mathrm{FUV}}-M_{\mathrm{NUV}}\right)-0.0088$ & $(42.25 \pm 0.04)+(0.90 \pm 0.03) \times\left(\log L_{\mathrm{H} \alpha, \text { Corr }}-40\right)$ & 0.29 & 0.29 & 0.06 & 62 \\
\hline $\log L_{8 \mu \mathrm{m}}$ & $(40.88 \pm 0.07)+(1.30 \pm 0.05) \times\left(\log L_{\mathrm{H} \alpha, \text { Corr }}-40\right)$ & 0.33 & 0.37 & 0.07 & 60 \\
\hline $\log L_{W 3}$ & $(40.79 \pm 0.06)+(1.27 \pm 0.04) \times\left(\log L_{\mathrm{H} \alpha, \text { Corr }}-40\right)$ & 0.28 & 0.34 & 0.05 & 61 \\
\hline $\log L_{W 4}$ & $(40.96 \pm 0.04)+(1.26 \pm 0.03) \times\left(\log L_{\mathrm{H} \alpha, \text { Corr }}-40\right)$ & 0.20 & 0.27 & 0.05 & 58 \\
\hline $\log L_{24 \mu \mathrm{m}}$ & $(40.93 \pm 0.04)+(1.30 \pm 0.03) \times\left(\log L_{\mathrm{H} \alpha, \text { Corr }}-40\right)$ & 0.18 & 0.24 & 0.08 & 62 \\
\hline $\log P_{1.4 \mathrm{GHz}}$ & $(19.65 \pm 0.05)+(1.27 \pm 0.03) \times\left(\log L_{\mathrm{H} \alpha, \text { Corr }}-40\right)$ & 0.18 & 0.22 & 0.08 & 52 \\
\hline $\log P_{150 \mathrm{MHz}}$ & $(20.49 \pm 0.08)+(1.16 \pm 0.05) \times\left(\log L_{\mathrm{H} \alpha, \text { Corr }}-40\right)$ & 0.24 & 0.32 & 0.08 & 36 \\
\hline $\log L_{8 \mu \mathrm{m}}$ & $(40.49 \pm 0.08)+\left(\log L_{\mathrm{H} \alpha, \text { Corr }}-40\right)+(0.38 \pm 0.04) \times\left(\log L_{4.5 \mu \mathrm{m}}-40\right)$ & 0.35 & 0.36 & 0.05 & 60 \\
\hline $\log L_{W 3}$ & $(40.52 \pm 0.05)+\left(\log L_{\mathrm{H} \alpha, \text { Corr }}-40\right)+(0.31 \pm 0.03) \times\left(\log L_{W 2}-40\right)$ & 0.25 & 0.29 & 0.05 & 61 \\
\hline $\log L_{W 4}$ & $(40.79 \pm 0.05)+\left(\log L_{\mathrm{H} \alpha, \text { Corr }}-40\right)+(0.25 \pm 0.02) \times\left(\log L_{W 2}-40\right)$ & 0.23 & 0.30 & 0.03 & 58 \\
\hline $\log L_{24 \mu \mathrm{m}}$ & $(40.69 \pm 0.05)+\left(\log L_{\mathrm{H} \alpha, \text { Corr }}-40\right)+(0.29 \pm 0.03) \times\left(\log L_{4.5} \mu \mathrm{m}-40\right)$ & 0.26 & 0.30 & 0.02 & 62 \\
\hline $\log P_{1.4 \mathrm{GHz}}$ & $(19.65 \pm 0.05)+\left(\log L_{\mathrm{H} \alpha, \text { Corr }}-40\right)+(0.27 \pm 0.03) \times\left(\log L_{W 2}-40\right)$ & 0.22 & 0.27 & 0.08 & 52 \\
\hline $\log P_{150 \mathrm{MHz}}$ & $(20.49 \pm 0.08)+\left(\log L_{\mathrm{H} \alpha, \text { Corr }}-40\right)+(0.16 \pm 0.05) \times\left(\log L_{W 2}-40\right)$ & 0.28 & 0.37 & 0.08 & 36 \\
\hline
\end{tabular}

Notes.

${ }^{\mathrm{a}} \mathrm{UV}$ and mid-infrared luminosities are presented in units of $\mathrm{erg} \mathrm{s}^{-1}$, while radio powers are presented in units of $\mathrm{W} \mathrm{Hz}^{-1}$

b $\sigma_{\mathrm{H} \alpha, \text { More }}$ is measured using galaxies that meet the less conservative BPT criterion of Kewley et al. (2001), which may include some AGNs that inflate the scatter. 

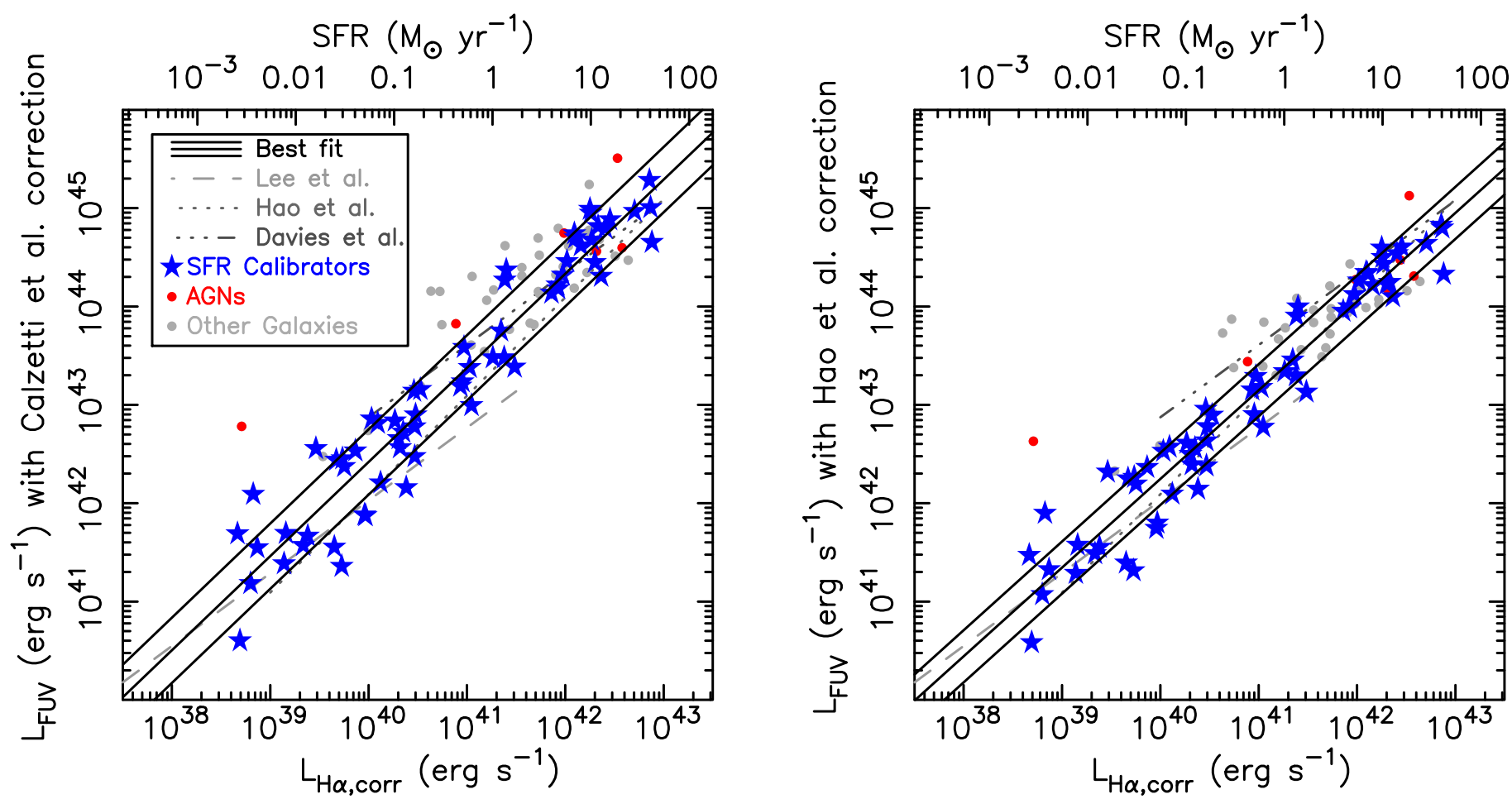

Figure 8. Dust-obscuration-corrected GALEX FUV luminosity as a function of Balmer-decrement-corrected H $\alpha$ luminosity, with Calzetti et al. (2000) and Hao et al. (2011) corrections for dust obscuration (derived from the observed $M_{\mathrm{FUV}}-M_{\mathrm{NUV}}$ ) used in the left and right panels, respectively. A STARBURST99 (Leitherer et al. 1999) model for a $100 \mathrm{Myr}$ old stellar population with Kroupa IMF (Hao et al. 2011) is comparable to the fits to our data. Although the power-law indices are within $10 \%$ of the expected value of one, the scatter of the data around the fits is $\sim 0.3$ dex for both panels.
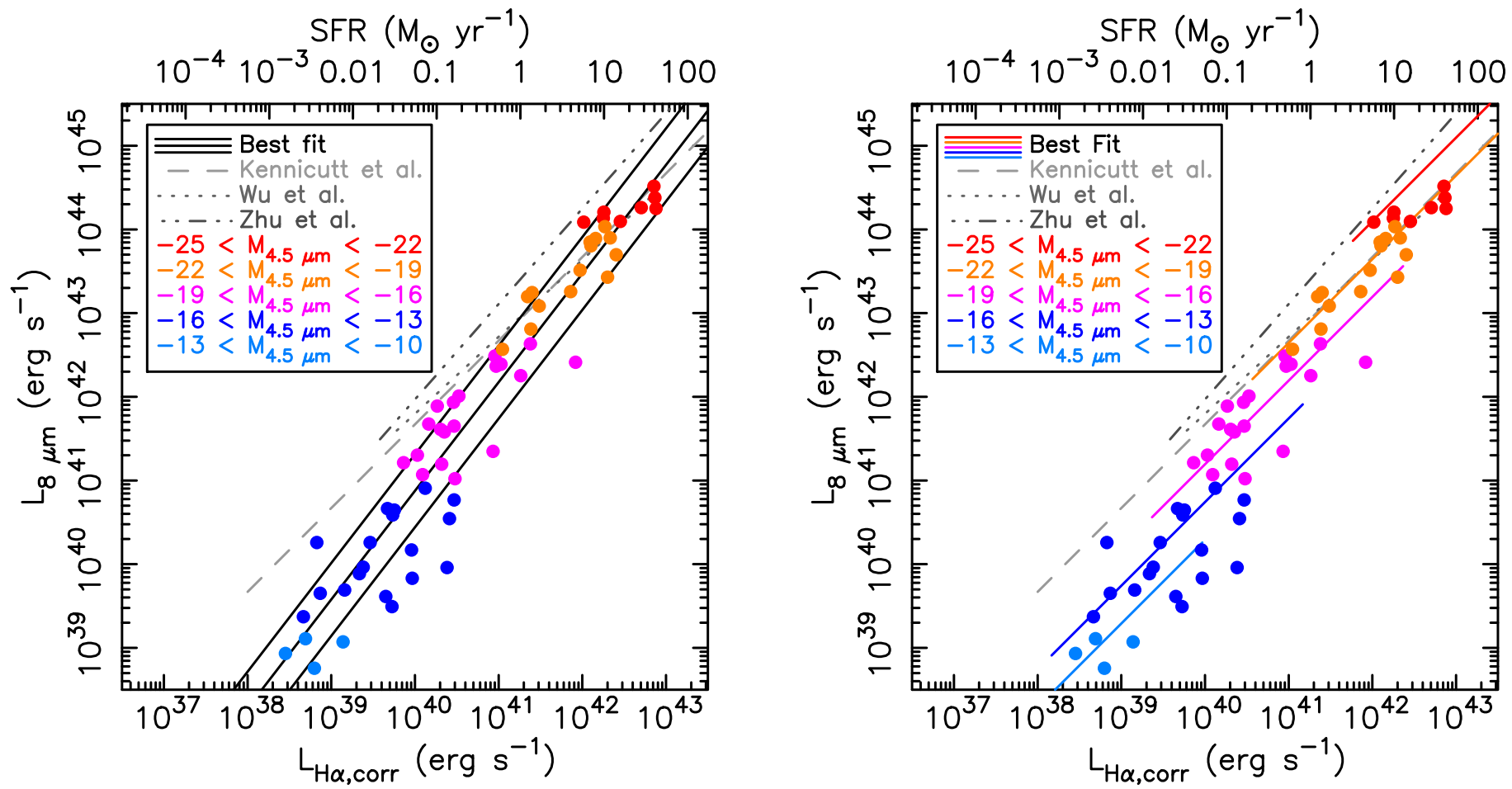

Figure 9. Spitzer $8 \mu \mathrm{m}$ luminosity as a function of Balmer-decrement-corrected $\mathrm{H} \alpha$, with data points color-coded by $4.5 \mu \mathrm{m}$ absolute magnitude (a rough stellar mass proxy). In the left panel, we plot a power-law fit to the data, while in the right panel, we plot a fit where the $8 \mu$ m luminosity scales linearly with the SFR and the normalization is a function of the $4.5 \mu \mathrm{m}$ luminosity. Although our power-law fit has an index of $1.30 \pm 0.05$, power laws from previous literature have indices closer to one.

In Figures 9-12, the data points are color-coded by $\sim 4.5 \mu \mathrm{m}$ luminosity, which is a rough proxy for stellar mass. The luminosity-luminosity correlations present in the sample are clearly evident, and suggest that the power-law fit parameters could depend on the mass range of the relevant calibration sample. Indeed, if we restrict our SFR calibrations to galaxies with $M_{4.5 \mu \mathrm{m}}<-17$, the power-law indices for the 8 and $24 \mu \mathrm{m}$ relations decrease to $1.10 \pm 0.05$ and $1.19 \pm 0.05$, 

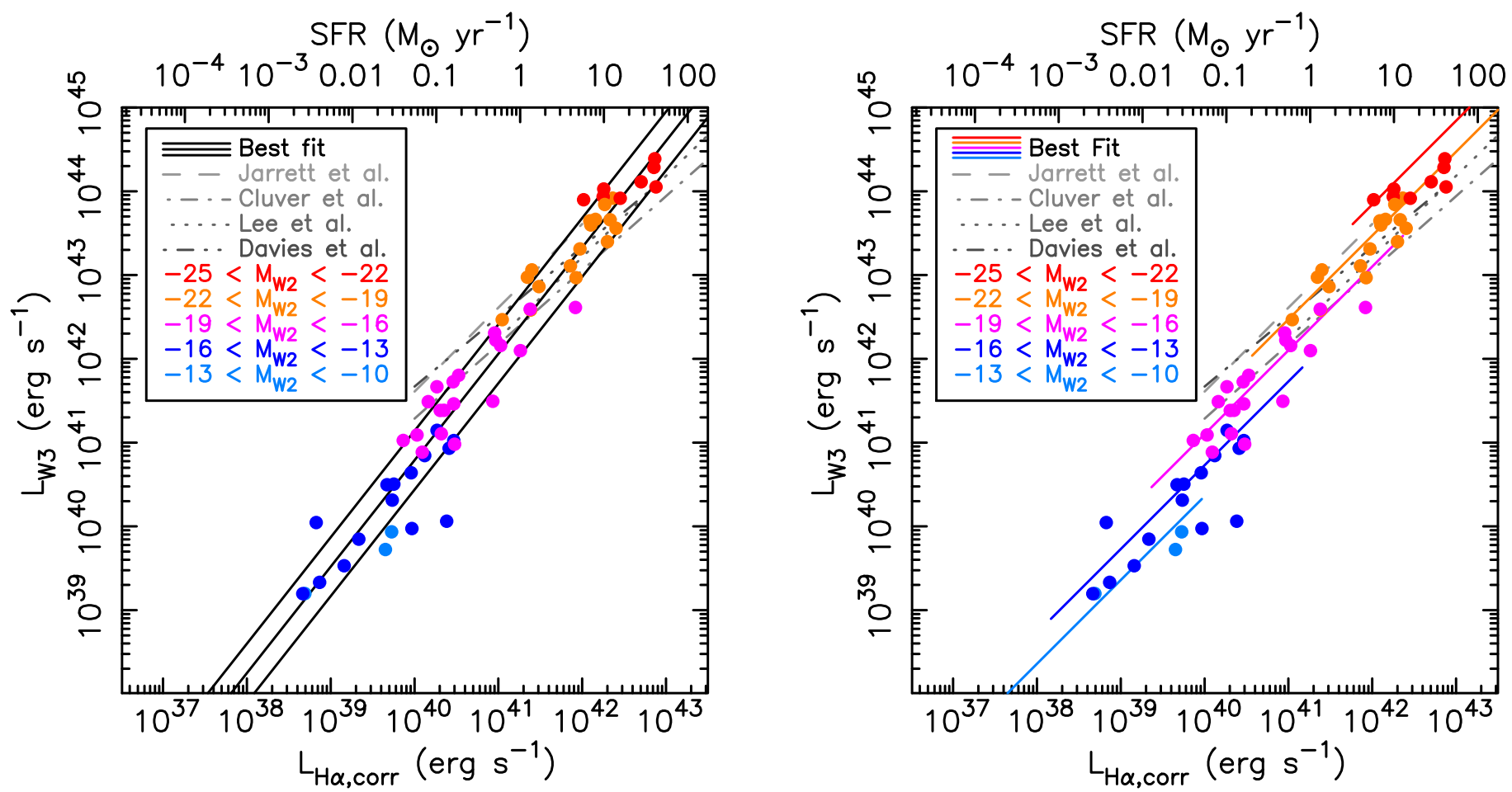

Figure 10. WISE W3 luminosity as a function of Balmer-decrement-corrected $\mathrm{H} \alpha$. For dwarf galaxies, we measure systematically higher $\mathrm{H} \alpha$ luminosities and SFRs at fixed W3 luminosity relative to extrapolations of relations from previous literature.
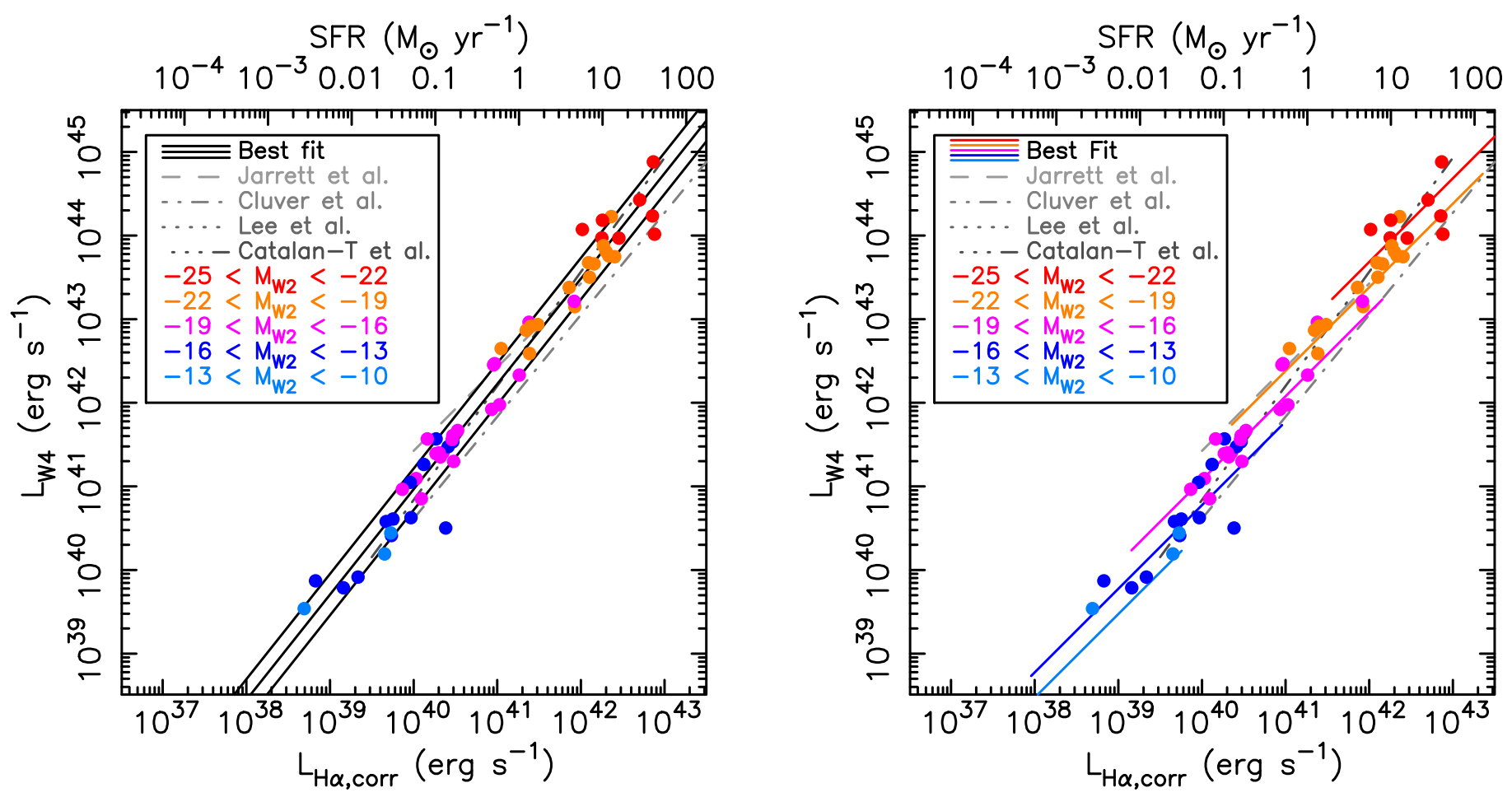

Figure 11. WISE W4 luminosity as a function of Balmer-decrement-corrected $\mathrm{H} \alpha$. Although the index of the power-law fit (left panel) is comparable to power-law fits to Spitzer $8 \mu \mathrm{m}$ and WISE W3 $(12 \mu \mathrm{m})$ data, the scatter around the best-fit relation is significantly reduced.

respectively, which is closer to the values in some previous literature. The dependence of the power-law indices on the stellar mass range of the sample flags a weakness of the powerlaw parameterization.

Our alternative to a power-law parameterization assumes that the SFR indicator luminosity scales linearly with SFR, with the normalization being a function of Spitzer $4.5 \mu \mathrm{m}$ or WISE W2 luminosity. Fits of this relation to the mid-infrared data are shown in the right-hand panels of Figures 9-12, and the fit parameters are presented in Table 4 . Effectively by construction, this parameterization agrees better with much of the literature for high-mass galaxies, where the power-law indices (both measured and adopted) are close to one. However, the scatter of the data about the fits using this parameterization are 

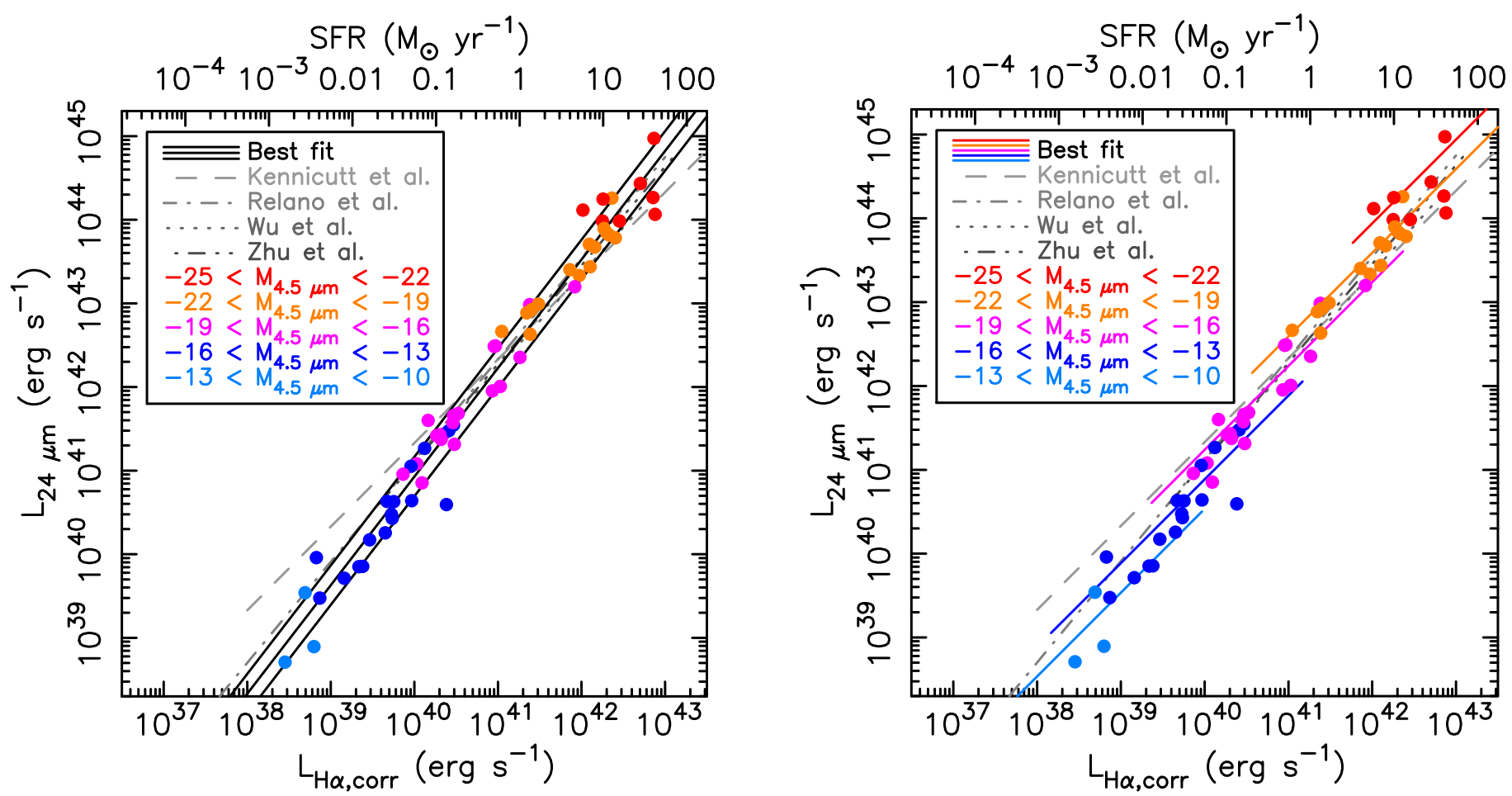

Figure 12. Spitzer $24 \mu \mathrm{m}$ luminosity as a function of Balmer-decrement-corrected $\mathrm{H} \alpha$, along with best-fit relations from previous literature (Wu et al. 2005; Relaño et al. 2007; Zhu et al. 2008; Kennicutt et al. 2009). Compared to the relations for Spitzer $8 \mu \mathrm{m}$ and W3, there is better agreement between our calibration and those from previous literature, although we still see offsets for the lowest-luminosity galaxies.

(marginally) worse than the scatter of the data about the powerlaw fits. Thus, on the basis of the data presented in this paper alone, there is no compelling reason to use this parameterization in preference over a power law, despite its potential aesthetic appeal.

\subsection{Radio Continuum}

We determined radio-continuum SFR calibrations at $1.4 \mathrm{GHz}$ and $150 \mathrm{MHz}$, which correspond to the frequencies of existing and planned wide-field radio-continuum surveys from the Karl G. Jansky Very Large Array, Low Frequency Array (LOFAR), Murchison Wide-field Array (MWA), and Australian Square Kilometre Array Pathfinder (ASAKP). Although the relationship between the $150 \mathrm{MHz}$ luminosity and far-infrared luminosity has been studied previously (e.g., Cox et al. 1988), our work is one of the first direct calibrations of $150 \mathrm{MHz}$ as an SFR indicator (e.g., Calistro Rivera et al. 2017; G. Gürkan et al. 2017, in preparation). Radiocontinuum emission from star-forming galaxies is dominated by thermal bremsstrahlung and non-thermal synchrotron components. As bremsstrahlung and synchrotron emission are expected to have spectra with (roughly) $f_{\nu} \propto \nu^{-0.1}$ and $f_{\nu} \propto \nu^{-0.7}$, respectively, synchrotron emission should be increasingly dominant at longer wavelengths. Synchrotron emission is dominant at $1.4 \mathrm{GHz}$ in $\sim L^{*}$ galaxies, but synchrotron emission depends on cosmic-ray production, magnetic field strength, and galaxy size (e.g., Bell 2003 and references therein), so the bremsstrahlung component is increasingly important with decreasing galaxy mass. Consequently, we do not expect radio luminosity to be directly proportional to SFR.

In Figures 13 and 14, we present the relationship between the $1.4 \mathrm{GHz}$ and $150 \mathrm{MHz}$ (respectively) radio-continuum

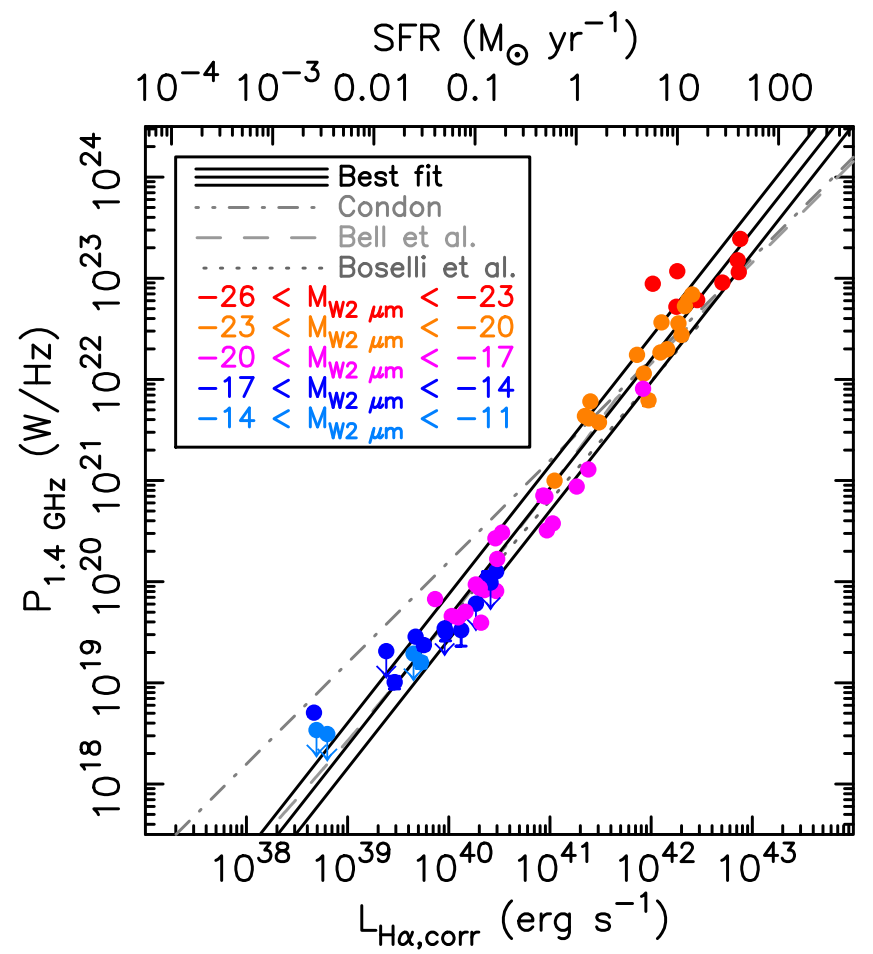

Figure 13. $1.4 \mathrm{GHz}$ continuum luminosity as a function of Balmer-decrementcorrected $\mathrm{H} \alpha$, along with relations from previous literature (Condon 1992; Bell 2003; Boselli et al. 2015). The scatter of the data around our best-fit power law is less than 0.2 dex. At low radio luminosities, we measure consistently higher $\mathrm{H} \alpha$ luminosities, and thus star formation rates, than the prior literature.

power and Balmer-decrement-corrected $\mathrm{H} \alpha$ luminosity. When fitting relations to the data, we only used radio sources with $>3 \sigma$ flux density measurements, but in Figures 13 and 14, we 


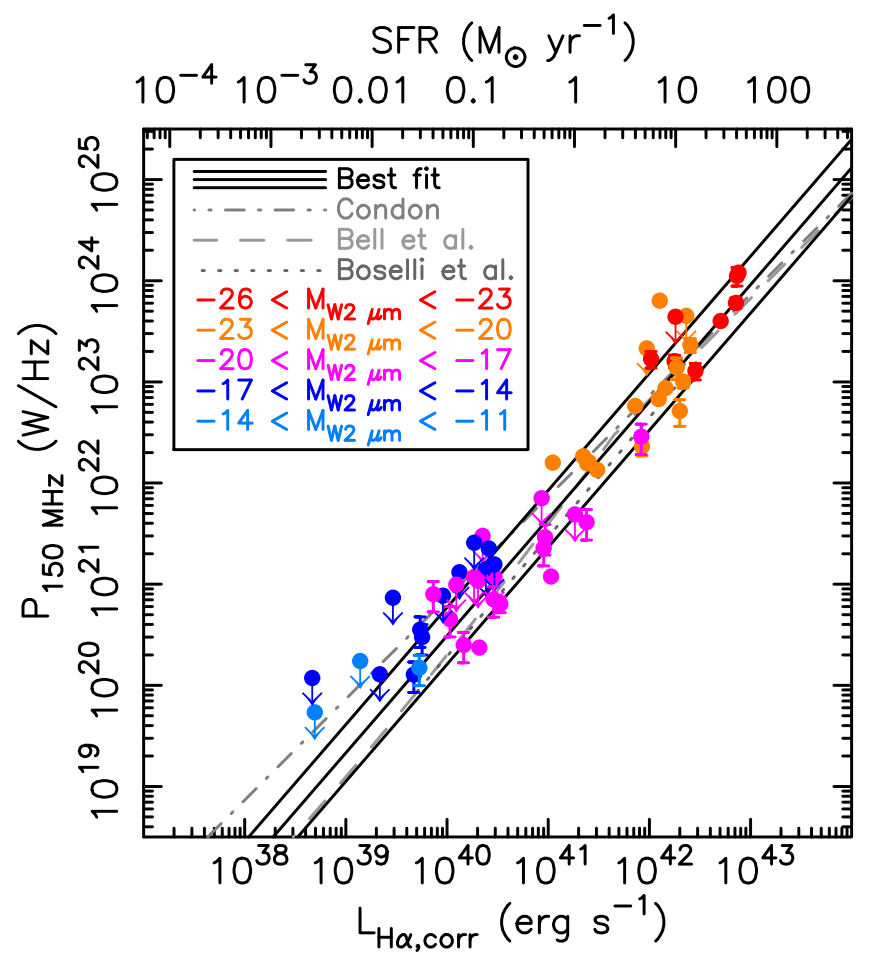

Figure 14. $150 \mathrm{MHz}$ continuum luminosity as a function of Balmer-decrementcorrected $\mathrm{H} \alpha$ luminosity. To plot relations from previous literature (Condon 1992; Bell 2003; Boselli et al. 2015), we extrapolated radio luminosities from $1.4 \mathrm{GHz}$ to $150 \mathrm{MHz}$ by assuming $f_{\nu} \propto \nu^{-0.7}$. Despite changing an order of magnitude in wavelength, our best-fit power law and the scatter of the data around this power law are comparable to those measured at $1.4 \mathrm{GHz}$.

also plot these upper limits. At $1.4 \mathrm{GHz}$, we find a power-law index of $1.27 \pm 0.03$ and a scatter of just $0.18 \mathrm{dex}$, which is comparable to the $24 \mu \mathrm{m}$ calibration. At $150 \mathrm{MHz}$, we find a shallower power-law index of $1.16 \pm 0.05$ and a scatter of 0.24 dex. Our alternative parameterization (not plotted) performs no better than the power-law parameterization, with marginally worse scatter for both $1.4 \mathrm{GHz}$ and $150 \mathrm{MHz}$.

Fits to the relationship between the radio-continuum luminosity and $\mathrm{H} \alpha$ luminosity from previous literature are also plotted in Figures 13 and 14. As there are no $150 \mathrm{MHz}$ versus $\mathrm{H} \alpha$ relations in the previous literature, we extrapolated $1.4 \mathrm{GHz}$ calibrations to $150 \mathrm{MHz}$ by assuming $f_{\nu} \propto \nu^{-0.7}$. Relative to the mid-infrared relations, there is generally better agreement between power-law fits from the prior literature and our work. This agreement may result from the power-law indices of the radio-continuum calibrations not being a strong function of the $\mathrm{H} \alpha$ luminosity and the sample stellar mass ranges. For example, when we restricted our calibrations to $M_{4.5 \mu \mathrm{m}}<-17$ galaxies, the power-law indices did not become significantly shallower.

\section{Summary}

We calibrated commonly used SFR indicators, including GALEX ultraviolet, Spitzer mid-infrared bands, and WISE midinfrared bands and radio continuum. This includes one of the first direct calibrations of $150 \mathrm{MHz}$ as an SFR indicator, which will be of use for new LOFAR and MWA wide-field surveys. The calibrations utilize 66 star-forming galaxies, including galaxies drawn from the Brown et al. (2014b) SED atlas and galaxies with distances less than $10 \mathrm{Mpc}$ with spectroscopy from Moustakas \& Kennicutt (2006) and Moustakas et al. (2010). Our sample includes a broad range of galaxy types and has absolute magnitudes of $-24<M_{r}<-12$ and colors of $0.0<M_{u}-M_{r}<2.3$. The sample also spans five orders of magnitude in $\mathrm{H} \alpha$ luminosity, which is broader than much of the previous literature, and we thus provide improved calibrations of SFR indicators for dwarf galaxies. Systematic errors associated with aperture corrections were mitigated by measuring ultraviolet and mid-infrared photometry with apertures matched to the same region as the spectrophotometry. To simplify transparency and reproducibility, all of the calibrations are anchored to Balmer-decrement-corrected $\mathrm{H} \alpha$ luminosities, assuming 10,000 K Case B recombination and a Fitzpatrick (1999) dust attenuation curve.

Our calibrations of SFR indicators are similar to those from the prior literature for $L^{*}$ galaxies, but for dwarf galaxies, we often find that (for fixed broadband luminosity) SFRs are higher than what one would expect using (extrapolated) relations from previous literature. We used two parameterizations to model the data, including the commonly used powerlaw relation and a linear relation where the normalization is a function of $4.5 \mu \mathrm{m}$ luminosity (a rough stellar mass proxy). We find that the power-law parameterization provides better fits to the data, although there is no expectation that galaxies with the same SFR but different stellar masses and metallicities should have the same SFR indicator luminosity. Scatter of the data around the best-fit relations is a function of wavelength, with the $1 \sigma$ scatter being only 0.2 dex for power-law fits to the WISE W4 $(22.8 \mu \mathrm{m})$, Spitzer $24 \mu \mathrm{m}$, and VLA $1.4 \mathrm{GHz}$ bands. We find that $150 \mathrm{MHz}$ is only slightly worse than $1.4 \mathrm{GHz}$ as an SFR indicator, with the data having only a 0.24 dex scatter about the best-fit power law for radio power as a function of $\mathrm{H} \alpha$ luminosity.

M.J.I.B. acknowledges financial support from The Australian Research Council (FT100100280), the Monash Research Accelerator Program (MRA), the Monash Outside Studies Programme (OSP), and the University of Cambridge. Part of this work was undertaken while M.J.I.B. was on OSP (sabbatical) leave at the University of Cambridge, Swinburne University, and the University of Melbourne. M.B. was supported by the MINEDUC-UA project, code ANT 1655.

This work is based in part on observations made with the Spitzer Space Telescope, obtained from the NASA/IPAC Infrared Science Archive, both of which are operated by the Jet Propulsion Laboratory, California Institute of Technology, under a contract with the National Aeronautics and Space Administration. This publication makes use of data products from the Wide-field Infrared Survey Explorer, which is a joint project of the University of California, Los Angeles, and the Jet Propulsion Laboratory/California Institute of Technology, funded by the National Aeronautics and Space Administration. We gratefully acknowledge NASA's support for construction, operation, and science analysis for the GALEX mission, developed in cooperation with the Centre National d'Etudes Spatiales of France and the Korean Ministry of Science and Technology.

The National Radio Astronomy Observatory is a facility of the National Science Foundation operated under cooperative agreement by Associated Universities, Inc. This research is based in part on observations taken with the telescopes of the 
National Optical Astronomy Observatory, which is operated by the Association of Universities for Research in Astronomy (AURA) under cooperative agreement with the National Science Foundation. We would like to thank the TIFR GMRT Sky Survey team for planning and carrying out the original observations, and we offer special thanks to the GMRT staff for their ongoing support. GMRT is run by the National Centre for Radio Astrophysics of the Tata Institute of Fundamental Research.

Funding for SDSS-III has been provided by the Alfred P. Sloan Foundation, the Participating Institutions, the National Science Foundation, and the U.S. Department of Energy Office of Science. The SDSS-III Web site is http://www.sdss3.org/. SDSS-III is managed by the Astrophysical Research Consortium for the Participating Institutions of the SDSS-III Collaboration including the University of Arizona, the Brazilian Participation Group, Brookhaven National Laboratory, University of Cambridge, University of Florida, the French Participation Group, the German Participation Group, the Instituto de Astrofisica de Canarias, the Michigan State/Notre Dame/JINA Participation Group, Johns Hopkins University, Lawrence Berkeley National Laboratory, Max Planck Institute for Astrophysics, New Mexico State University, New York University, Ohio State University, Pennsylvania State University, University of Portsmouth, Princeton University, the Spanish Participation Group, University of Tokyo, University of Utah, Vanderbilt University, University of Virginia, University of Washington, and Yale University. The NASA-Sloan Atlas was created by Michael Blanton, with extensive help and testing from Eyal Kazin, Guangtun Zhu, Adrian Price-Whelan, John Moustakas, Demitri Muna, Renbin Yan, and Benjamin Weaver. Funding for the NASA-Sloan Atlas has been provided by the NASA Astrophysics Data Analysis Program (08-ADP08-0072) and the NSF (AST-1211644).

Facilities: Bok (Boller \& Chivens spectrograph), CTIO:1.5m (R-C spectrograph), GMRT, Sloan, Spitzer (IRAC, IRS, MIPS), VLA, WISE.

\section{ORCID iDs}

Michael J. I. Brown (다 https://orcid.org/0000-0002-1207-9137 Nicolas J. Bonne (1) https://orcid.org/0000-0002-1165-6801 Huib T. Intema (1) https://orcid.org/0000-0002-5880-2730 Francesco de Gasperin (1) https://orcid.org/0000-00034439-2627

Mederic Boquien (1) https://orcid.org/0000-0003-0946-6176 T. H. Jarrett (1) https://orcid.org/0000-0002-4939-734X Michelle E. Cluver (i) https://orcid.org/0000-0002-9871-6490 J.-D. T. Smith (1) https://orcid.org/0000-0003-1545-5078 Elisabete da Cunha (i) https://orcid.org/0000-0001-9759-4797 Masatoshi Imanishi (ㄷ) https://orcid.org/0000-0001-6186-8792 J. E. G. Peek (1) https://orcid.org/0000-0003-4797-7030

\section{References}

Aihara, H., Allende Prieto, C., An, D., et al. 2011, ApJS, 193, 29 Baars, J. W. M., Genzel, R., Pauliny-Toth, I. I. K., \& Witzel, A. 1977, A\&A, 61, 99 Bagchi, J., Sirothia, S. K., Werner, N., et al. 2011, ApJL, 736, L8 Baldry, I. K., \& Glazebrook, K. 2003, ApJ, 593, 258 Baldwin, J. A., Phillips, M. M., \& Terlevich, R. 1981, PASP, 93, 5 Baldwin, J. E., Boysen, R. C., Hales, S. E. G., et al. 1985, MNRAS, 217, 717 Bell, E. F. 2003, ApJ, 586, 794

Bendo, G. J., Wilson, C. D., Pohlen, M., et al. 2010, A\&A, 518, L65

Blanton, M. R., Kazin, E., Muna, D., Weaver, B. A., \& Price-Whelan, A. 2011, AJ, 142, 31

Bohlin, R. C., Gordon, K. D., Rieke, G. H., et al. 2011, AJ, 141, 173
Bohlin, R. C., Gordon, K. D., \& Tremblay, P.-E. 2014, PASP, 126, 711 Boquien, M., Buat, V., Boselli, A., et al. 2012, A\&A, 539, A145 Boquien, M., Calzetti, D., Combes, F., et al. 2011, AJ, 142, 111 Boquien, M., Kennicutt, R., Calzetti, D., et al. 2016, A\&A, 591, A6 Boselli, A., Fossati, M., Gavazzi, G., et al. 2015, A\&A, 579, A102 Brough, S., Hopkins, A. M., Sharp, R. G., et al. 2011, MNRAS, 413, 1236 Brown, M. J. I., Jarrett, T. H., \& Cluver, M. E. 2014a, PASA, 31, 49 Brown, M. J. I., Moustakas, J., Smith, J.-D. T., et al. 2014b, ApJS, 212, 18 Bruzual, G., \& Charlot, S. 2003, MNRAS, 344, 1000

Calistro Rivera, G., Williams, W. L., Hardcastle, M. J., et al. 2017, MNRAS, 469, 3468

Calzetti, D., Armus, L., Bohlin, R. C., et al. 2000, ApJ, 533, 682

Calzetti, D., Kennicutt, R. C., Engelbracht, C. W., et al. 2007, ApJ, 666, 870

Calzetti, D., Kinney, A. L., \& Storchi-Bergmann, T. 1994, ApJ, 429, 582

Camarota, L., \& Holberg, J. B. 2014, MNRAS, 438, 3111

Cappellari, M., \& Emsellem, E. 2004, PASP, 116, 138

Catalán-Torrecilla, C., Gil de Paz, A., Castillo-Morales, A., et al. 2015, A\&A, 584, A87

Chabrier, G. 2003, PASP, 115, 763

Charlot, S., \& Fall, S. M. 2000, ApJ, 539, 718

Cluver, M. E., Jarrett, T. H., Hopkins, A. M., et al. 2014, ApJ, 782, 90

Condon, J. J. 1992, ARA\&A, 30, 575

Condon, J. J., Cotton, W. D., \& Broderick, J. J. 2002, AJ, 124, 675

Condon, J. J., Cotton, W. D., Greisen, E. W., et al. 1998, AJ, 115, 1693

Cortés, J. R., Kenney, J. D. P., \& Hardy, E. 2008, ApJ, 683, 78

Cox, M. J., Eales, S. A. E., Alexander, P., \& Fitt, A. J. 1988, MNRAS, 235, 1227 da Cunha, E., Charlot, S., \& Elbaz, D. 2008, MNRAS, 388, 1595

da Silva, R. L., Fumagalli, M., \& Krumholz, M. R. 2014, MNRAS, 444, 3275 Davies, L. J. M., Driver, S. P., Robotham, A. S. G., et al. 2016, MNRAS, 461, 458

Dopita, M. A., \& Sutherland, R. S. 2003, Astrophysics of the Diffuse Universe (Berlin: Springer)

Draine, B. T., Dale, D. A., Bendo, G., et al. 2007, ApJ, 663, 866

Elbaz, D., Dickinson, M., Hwang, H. S., et al. 2011, A\&A, 533, A119

Engelbracht, C. W., Blaylock, M., Su, K. Y. L., et al. 2007, PASP, 119, 994 Engelbracht, C. W., Gordon, K. D., Rieke, G. H., et al. 2005, ApJL, 628, L29 Engelbracht, C. W., Rieke, G. H., Gordon, K. D., et al. 2008, ApJ, 678, 804 Fazio, G. G., Hora, J. L., Allen, L. E., et al. 2004, ApJS, 154, 10

Fitzpatrick, E. L. 1999, PASP, 111, 63

Gil de Paz, A., Boissier, S., Madore, B. F., et al. 2007, ApJS, 173, 185

Gopal-Krishna, Mhaskey, M., Wiita, P. J., et al. 2012, MNRAS, 423, 1053

Goto, T., Arnouts, S., Inami, H., et al. 2011, MNRAS, 410, 573

Hales, S. E. G., Baldwin, J. E., \& Warner, P. J. 1988, MNRAS, 234, 919

Hales, S. E. G., Baldwin, J. E., \& Warner, P. J. 1993a, MNRAS, 263, 25

Hales, S. E. G., Masson, C. R., Warner, P. J., \& Baldwin, J. E. 1990, MNRAS, 246, 256

Hales, S. E. G., Masson, C. R., Warner, P. J., Baldwin, J. E., \& Green, D. A. 1993b, MNRAS, 262, 1057

Hales, S. E. G., Mayer, C. J., Warner, P. J., \& Baldwin, J. E. 1991, MNRAS, 251, 46

Hales, S. E. G., Riley, J. M., Waldram, E. M., Warner, P. J., \& Baldwin, J. E. 2007, MNRAS, 382, 1639

Hao, C.-N., Kennicutt, R. C., Johnson, B. D., et al. 2011, ApJ, 741, 124

Hopkins, A. M., Miller, C. J., Nichol, R. C., et al. 2003, ApJ, 599, 971

Houck, J. R., Charmandaris, V., Brandl, B. R., et al. 2004, ApJS, 154, 211

Hummer, D. G., \& Storey, P. J. 1987, MNRAS, 224, 801

Hurley-Walker, N., Callingham, J. R., Hancock, P. J., et al. 2017, MNRAS, 464, 1146

Imanishi, M., Nakagawa, T., Shirahata, M., Ohyama, Y., \& Onaka, T. 2010, ApJ, 721, 1233

Intema, H. T., Jagannathan, P., Mooley, K. P., \& Frail, D. A. 2017, A\&A, 598, A78

Jackson, D. C., Cannon, J. M., Skillman, E. D., et al. 2006, ApJ, 646, 192

Jaiswal, S., \& Omar, A. 2016, MNRAS, 462, 92

Jarrett, T. H., Cohen, M., Masci, F., et al. 2011, ApJ, 735, 112

Jarrett, T. H., Masci, F., Tsai, C. W., et al. 2013, AJ, 145, 6

Kauffmann, G., Heckman, T. M., Tremonti, C., et al. 2003, MNRAS, 346, 1055

Kennicutt, R. C., \& Evans, N. J. 2012, ARA\&A, 50, 531

Kennicutt, R. C., Jr., Armus, L., Bendo, G., et al. 2003, PASP, 115, 928

Kennicutt, R. C., Jr., Hao, C.-N., Calzetti, D., et al. 2009, ApJ, 703, 1672

Kennicutt, R. C., Jr., Lee, J. C., Funes, J. G., et al. 2008, ApJS, 178, 247

Kewley, L. J., Dopita, M. A., Sutherland, R. S., Heisler, C. A., \& Trevena, J. 2001, ApJ, 556, 121

Kroupa, P. 2001, MNRAS, 322, 231

Lee, J. C., Gil de Paz, A., Tremonti, C., et al. 2009, ApJ, 706, 599 
Lee, J. C., Hwang, H. S., \& Ko, J. 2013, ApJ, 774, 62

Leitherer, C., Schaerer, D., Goldader, J. D., et al. 1999, ApJS, 123, 3

Leja, J., Johnson, B. D., Conroy, C., van Dokkum, P. G., \& Byler, N. 2017, ApJ, 837, 170

Lisker, T., \& Han, Z. 2008, ApJ, 680, 1042

Marconi, M., Musella, I., Fiorentino, G., et al. 2010, ApJ, 713, 615

Morrissey, P., Conrow, T., Barlow, T. A., et al. 2007, ApJS, 173, 682

Mould, J. R., Huchra, J. P., Freedman, W. L., et al. 2000, ApJ, 529, 786

Moustakas, J., \& Kennicutt, R. C., Jr. 2006, ApJS, 164, 81

Moustakas, J., Kennicutt, R. C., Jr., Tremonti, C. A., et al. 2010, ApJS, 190, 233

Moustakas, J., Zaritsky, D., Brown, M., et al. 2011, arXiv:1112.3300

Noeske, K. G., Weiner, B. J., Faber, S. M., et al. 2007, ApJL, 660, L43

Noll, S., Burgarella, D., Giovannoli, E., et al. 2009, A\&A, 507, 1793

Norris, R. P., Hopkins, A. M., Afonso, J., et al. 2011, PASA, 28, 215

O'Donnell, J. E. 1994, ApJ, 422, 158

Padmanabhan, N., Schlegel, D. J., Finkbeiner, D. P., et al. 2008, ApJ, 674, 1217

Papovich, C., Moustakas, L. A., Dickinson, M., et al. 2006, ApJ, 640, 92

Peek, J. E. G. 2013, arXiv:1311.2941

Planck Collaboration, Ade, P. A. R., Aghanim, N., et al. 2011, A\&A, 536, A19

Planck Collaboration, Ade, P. A. R., Aghanim, N., et al. 2014, A\&A, 571, A1

Relaño, M., Lisenfeld, U., Pérez-González, P. G., Vílchez, J. M., \& Battaner, E. 2007, ApJL, 667, L141

Rieke, G. H., Alonso-Herrero, A., Weiner, B. J., et al. 2009, ApJ, 692, 556

Rieke, G. H., Young, E. T., Engelbracht, C. W., et al. 2004, ApJS, 154, 25
Roming, P. W. A., Kennedy, T. E., Mason, K. O., et al. 2005, SSRv, 120, 95 Rujopakarn, W., Rieke, G. H., Weiner, B. J., et al. 2013, ApJ, 767, 73

Salpeter, E. E. 1955, ApJ, 121, 161

Sarzi, M., Falcón-Barroso, J., Davies, R. L., et al. 2006, MNRAS, 366, 1151 Scaife, A. M. M., \& Heald, G. H. 2012, MNRAS, 423, L30

Sirothia, S. K., Lecavelier des Etangs, A., Gopal-Krishna, Kantharia, N. G., \& Ishwar-Chandra, C. H. 2014, A\&A, 562, A108

Skrutskie, M. F., Cutri, R. M., Stiening, R., et al. 2006, AJ, 131, 1163

Smith, J. D. T., Draine, B. T., Dale, D. A., et al. 2007, ApJ, 656, 770

Sorce, J. G., Tully, R. B., Courtois, H. M., et al. 2014, MNRAS, 444, 527

Stern, D., Eisenhardt, P., Gorjian, V., et al. 2005, ApJ, 631, 163

Storey, P. J., \& Hummer, D. G. 1995, MNRAS, 272, 41

The Dark Energy Survey Collaboration 2005, arXiv:astro-ph/0510346

Tremonti, C. A., Heckman, T. M., Kauffmann, G., et al. 2004, ApJ, 613 898

Tully, R. B., Courtois, H. M., Dolphin, A. E., et al. 2013, AJ, 146, 86

van Dokkum, P. G., \& Conroy, C. 2010, Natur, 468, 940

Walterbos, R. A. M., \& Schwering, P. B. W. 1987, A\&A, 180, 27

Wayth, R. B., Lenc, E., Bell, M. E., et al. 2015, PASA, 32, e025

Weisz, D. R., Johnson, B. D., Johnson, L. C., et al. 2012, ApJ, 744, 44

Williams, W. L., van Weeren, R. J., Röttgering, H. J. A., et al. 2016, MNRAS, 460,2385

Wright, E. L., Eisenhardt, P. R. M., Mainzer, A. K., et al. 2010, AJ, 140, 1868

Wu, H., Cao, C., Hao, C.-N., et al. 2005, ApJL, 632, L79

Wu, Y., Charmandaris, V., Hao, L., et al. 2006, ApJ, 639, 157

Zhu, Y.-N., Wu, H., Cao, C., \& Li, H.-N. 2008, ApJ, 686, 155 